\title{
DE BRANGES-ROVNYAK SPACES: BASICS AND THEORY
}

\author{
JOSEPH A. BALL AND VLADIMIR BOLOTNIKOV
}

\begin{abstract}
For $S$ a contractive analytic operator-valued function on the unit disk $\mathbb{D}$, de Branges and Rovnyak associate a Hilbert space of analytic functions $\mathcal{H}(S)$ and related extension space $\mathcal{D}(S)$ consisting of pairs of analytic functions on the unit disk $\mathbb{D}$. This survey describes three equivalent formulations (the original geometric de Branges-Rovnyak definition, the Toeplitz operator characterization, and the characterization as a reproducing kernel Hilbert space) of the de Branges-Rovnyak space $\mathcal{H}(S)$, as well as its role as the underlying Hilbert space for the modeling of completely non-isometric Hilbert-space contraction operators. Also examined is the extension of these ideas to handle the modeling of the more general class of completely nonunitary contraction operators, where the more general two-component de BrangesRovnyak model space $\mathcal{D}(S)$ and associated overlapping spaces play key roles. Connections with other function theory problems and applications are also discussed. More recent applications to a variety of subsequent applications are given in a companion survey article.
\end{abstract}

\section{Contents}

1. Introduction

2. The original de Branges-Rovnyak formulation

3. The de Branges-Rovnyak space $\mathcal{H}(S)$ : other formulations

3.1. Lifted-norm spaces

3.2. Pullback spaces

3.3. Spaces associated with Toeplitz operators $\quad 15$

3.4. Reproducing kernel Hilbert spaces $\quad 19$

4. The de Branges-Rovnyak space $\mathcal{D}(S)$

4.1. $\mathcal{D}(S)$ as a reproducing kernel Hilbert space $\quad 33$

4.2. $\mathcal{D}(S)$ as a pullback space 37

1991 Mathematics Subject Classification. 47A57.

Key words and phrases. de Branges-Rovnyak spaces, lifted norm, Brangesian complementary space, reproducing kernel Hilbert space. 
4.3. Two-component overlapping spaces: factorization and invariant subspaces

5. Generalizations and extensions

5.1. Canonical de Branges-Rovnyak functional-model spaces: multivariable settings

5.2. Extensions to KreĬn space settings 42

6. Concluding remarks 43

6.1. Still other settings $\quad 43$

6.2. Special questions 44

6.3. Applications $\quad 44$

References 44

\section{INTRODUCTION}

In the late 1960s and early 1970s, Louis de Branges and James Rovnyak introduced and studied spaces of vector-valued holomorphic functions on the open unit disk $\mathbb{D}$ associated with what is now called a Schur-class function $S \in \mathcal{S}(\mathcal{U}, \mathcal{Y})$ (i.e., a holomorphic function $S$ on the unit disk with values equal to contraction operators between Hilbert coefficient spaces $\mathcal{U}$ and $\mathcal{Y}$ - although in the original work of de Branges and Rovnyak the choice $\mathcal{U}=\mathcal{Y}$ was usually taken). These spaces were related to but distinct from the Hilbert spaces of entire functions explored in earlier work of de Branges (see in particular the book dB1968]); these latter spaces in turn have been revived recently, especially in the work of $\mathrm{H}$. Dym and associates (see DyMcK1976, AD2008, AD2012 ) as well as others and have deep connections with the work of M.G. Kreı̆n and assorted applied problems (e.g., continuous analogs of orthogonal polynomials and associated moment problems, inverse string problems). These spaces also serve as model spaces for unbounded densely defined symmetric operators with equal deficiency indices. As other authors will be discussing these spaces in other chapters of this series, our focus here will be on the de Branges-Rovnyak spaces on the unit disk. Motivation for the study of these spaces seems to be from at least two sources:

(1) quantum scattering theory (see dBR1966a as well as the papers dB1977, dBS1968]), and

(2) operator model theory for Hilbert space contraction operators and the invariant subspace problem (see [BR1966a, Appendix] and [BR1966b]). 
The connection with quantum scattering had to do with using the machinery of Hilbert spaces of analytic functions (in particular, an object called overlapping spaces) to set up a formalism for the study of the perturbation theory for self-adjoint operators (or equivalently after Cayley transformation, to the perturbation theory of unitary operators), an important topic in the wave-operator approach to scattering theory. This article does not go into this topic, but rather focuses on the second application, namely, to operator model theory.

There are now at least three distinct ways of introducing the de Branges-Rovnyak spaces:

(1) the original definition of de Branges and Rovnyak (as the complementary space of $S \cdot H^{2}$,

(2) as the range of the Toeplitz defect operator with lifted norm, or

(3) as the reproducing kernel Hilbert space with reproducing kernel given by the de Branges-Rovnyak positive kernel.

In the next three sections, each of these will be discussed in turn.

\section{The original DE Branges-Rovnyak FORMUlation}

In what follows, the symbol $\mathcal{L}(\mathcal{U}, \mathcal{Y})$ stands for the space of bounded linear operators mapping a Hilbert space $\mathcal{U}$ into a Hilbert space $\mathcal{Y}$, abbreviated to $\mathcal{L}(\mathcal{Y})$ in case $\mathcal{U}=\mathcal{Y}$. The standard Hardy space of $\mathcal{Y}$-valued functions on the open unit disk $\mathbb{D}$ with square-summable sequences of Taylor coefficient is denoted by $H^{2}(\mathcal{Y})$ and the notation $\mathcal{S}(\mathcal{U}, \mathcal{Y})$ is used for the Schur class of functions analytic on $\mathbb{D}$ whose values are contractive operators in $\mathcal{L}(\mathcal{U}, \mathcal{Y})$.

Let $S \in \mathcal{S}(\mathcal{U}, \mathcal{Y})$ be a Schur-class function. L. de Branges and J. Rovnyak define the space $\mathcal{H}(S)$ according to the prescription

$\mathcal{H}(S)=\left\{f \in H^{2}(\mathcal{U}):\|f\|_{\mathcal{H}(S)}^{2}:=\sup _{g \in H^{2}(\mathcal{U})}\left\{\|f+S g\|_{H^{2}(\mathcal{Y})}^{2}-\|g\|_{H^{2}(\mathcal{U})}^{2}\right\}<\infty\right\}$.

At first glance the definition looks rather impenetrable, except for one easy special case, namely, the case where $S$ is inner. In this case, it is relatively straightforward to see that $\mathcal{H}(S)$ is isometrically equal to $H^{2}(\mathcal{Y}) \ominus S \cdot H^{2}(\mathcal{U})$. Nevertheless, it is possible to show directly from the definition (2.1) (see dBR1966a, dBR1966b) the following basic facts listed in Theorem 2.1, the notion of reproducing kernel Hilbert space entering in the first fact is reviewed in Section 3.4 below. The proofs of the various pieces of the following result are given also in Section 3.4 . 
Theorem 2.1. If $S \in \mathcal{S}(\mathcal{U}, \mathcal{Y})$, the space $\mathcal{H}(S)$ has the following properties:

(1) $\mathcal{H}(S)$ is a linear space, indeed a reproducing kernel Hilbert space with reproducing kernel $K_{S}(z, w)$ given by

$$
K_{S}(z, w)=\frac{I-S(z) S(w)^{*}}{1-z \bar{w}} .
$$

(2) The space $\mathcal{H}(S)$ is invariant under the backward-shift operator

$$
R_{0}: f(z) \mapsto[f(z)-f(0)] / z
$$

and the following norm estimate holds:

$$
\left\|R_{0} f\right\|_{\mathcal{H}(S)}^{2} \leq\|f\|_{\mathcal{H}(S)}^{2}-\|f(0)\|_{\mathcal{Y}}^{2} .
$$

Moreover, equality holds in (2.3) for all $f \in \mathcal{H}(S)$ if and only if $\mathcal{H}(S)$ has the property

$$
S(z) \cdot u \in \mathcal{H}(S) \Rightarrow S(z) \cdot u \equiv 0 .
$$

(3) For any $u \in \mathcal{U}$, the function $R_{0}(S u)$ is in $\mathcal{H}(S)$. If one lets $\tau: \mathcal{U} \rightarrow \mathcal{H}(S)$ denote the operator

$$
\tau: u \mapsto R_{0}(S u)=\frac{S(z)-S(0)}{z} u
$$

then the adjoint $R_{0}^{*}$ of the operator $R_{0}(2.2)$ on $\mathcal{H}(S)$ is given by

$$
R_{0}^{*}: f(z) \mapsto z f(z)-S(z) \cdot \tau^{*}(f)
$$

with the following formula for the norm holding:

$$
\left\|R_{0}^{*} f\right\|_{\mathcal{H}(S)}^{2}=\|f\|_{\mathcal{H}(S)}^{2}-\left\|\tau^{*}(f)\right\|_{\mathcal{U}}^{2} .
$$

(4) Let $\mathbf{U}_{S}$ be the colligation matrix given by

$$
\mathbf{U}_{S}=\left[\begin{array}{cc}
A_{S} & B_{S} \\
C_{S} & D_{S}
\end{array}\right]:=\left[\begin{array}{cc}
R_{0} & \tau \\
\mathbf{e}(0) & S(0)
\end{array}\right]:\left[\begin{array}{c}
\mathcal{H}(S) \\
\mathcal{U}
\end{array}\right] \rightarrow\left[\begin{array}{c}
\mathcal{H}(S) \\
\mathcal{Y}
\end{array}\right]
$$

where $R_{0}$ and $\tau$ are given by (2.2) and (2.4) and where $\mathbf{e}(0): \mathcal{H}(S) \rightarrow$ $\mathcal{Y}$ is the evaluation-at-zero map:

$$
\mathbf{e}(0): f(z) \mapsto f(0) .
$$

Then $\mathbf{U}_{S}$ is coisometric, and one recovers $S(z)$ as the characteristic function of $\mathbf{U}_{S}$ :

$$
S(z)=D_{S}+z C_{S}\left(I-z A_{S}\right)^{-1} B_{S} .
$$


(5) The operator $T$ on a Hilbert space $\mathcal{X}$ is unitarily equivalent to an operator of the form $R_{0}$ on a model space $\mathcal{H}_{S}$ for a contractive operator-valued function $S$ on $\mathbb{D}$ if and only if $T$ is a completely non-isometric contraction, i.e.,

$$
\|T\| \leq 1 \quad \text { and } \bigcap_{n \geq 0}\left\{x:\left\|T^{n} x\right\|=\|x\|\right\}=\{0\} .
$$

In addition, there is an extended space $\mathcal{D}(S)$ constructed as follows (see dBR1966a, dB1970]). One defines $\mathcal{D}(S)$ as the space of all pairs of functions (here written as columns) $\left[\begin{array}{l}f(z) \\ g(z)\end{array}\right]$ with $f \in H^{2}(\mathcal{Y})$ and $\left.g(z)=\sum_{n=0}^{\infty} a_{n} z^{n} \in H^{2}(\mathcal{U})\right)$ such that the sequence of numbers $N_{n}:=\left\|z^{n} f(z)-S(z)\left(a_{0} z^{n-1}+\cdots+a_{n-1}\right)\right\|_{\mathcal{H}(S)}^{2}+\left\|a_{0}\right\|^{2}+\cdots+\left\|a_{n-1}\right\|^{2}$

is uniformly bounded. It can be shown that the sequence $\left\{N_{n}\right\}_{n \geq 0}$ is in fact nonincreasing so the limit $\lim _{n \rightarrow \infty} N_{n}$ exists. This limit is then defined to be the $\mathcal{D}(S)$-norm of $\left[\begin{array}{c}f(z) \\ g(z)\end{array}\right]$ :

$$
\left\|\left[\begin{array}{l}
f \\
g
\end{array}\right]\right\|_{\mathcal{D}(S)}^{2}=\lim _{n \rightarrow \infty} N_{n} \quad \text { where } N_{n} \text { is as in (2.9). }
$$

In particular, if $\left[\begin{array}{l}f \\ g\end{array}\right] \in \mathcal{D}(S)$, then necessarily

$$
z^{n} f(z)-S(z)\left(a_{0} z^{n-1}+\cdots+a_{n-1}\right) \in \mathcal{H}(S)
$$

for each $n=0,1,2, \ldots$ The special choice $n=0$ implies that $f \in$ $\mathcal{H}(S)$. The formula (2.5) for $R_{0}^{*}$ combined with the notation $a_{i}=$ $\tau^{*}\left(\left(R_{0}^{*}\right)^{i+1} f\right)$ gives rise to the formula

$$
z \mapsto z^{n} f(z)-S(z)\left(a_{0} z^{n-1}+\cdots+a_{n-1}\right)=\left(R_{0}^{*}\right)^{n}(f) \in \mathcal{H}(S)
$$

for the action of $\left(R_{0}^{*}\right)^{n}$. Moreover, the norm identity (2.6) implies that $\left\|z^{n} f(z)-S(z)\left(a_{0} z^{n-1}+\cdots+a_{n-1}\right)\right\|_{\mathcal{H}(S)}^{2}+\left\|a_{n-1}\right\|^{2}+\cdots+\left\|a_{0}\right\|^{2}=\|f(z)\|_{\mathcal{H}(S)}^{2}$ for all $n \geq 0$. For this special choice of $g$, namely

$$
g(z)=\widetilde{f}(z):=\sum_{n=0}^{\infty} a_{n} z^{n} \quad \text { with } \quad a_{n}=\tau^{*}\left(\left(R_{0}^{*}\right)^{n+1} f\right)
$$

it follows that $\left[\begin{array}{c}f \\ \widetilde{f}\end{array}\right] \in \mathcal{D}(S)$ with $\left\|\left[\begin{array}{l}f \\ \widetilde{f}\end{array}\right]\right\|_{\mathcal{D}(S)}=\|f\|_{\mathcal{H}(S)}$. Thus $f \mapsto\left[\begin{array}{l}f \\ \widetilde{f}\end{array}\right]$ is an isometric embedding of $\mathcal{H}(S)$ into $\mathcal{D}(S)$.

The following theorem gives the properties of $\mathcal{D}(S)$ analogous to those listed in Theorem 2.1 for $\mathcal{H}(S)$. The proofs of these results are given in Section 4.1 below. 
Theorem 2.2. Suppose that $S \in \mathcal{S}(\mathcal{U}, \mathcal{Y})$ and the space $\mathcal{D}(S)$ is defined as above. Then:

(1) $\mathcal{D}(S)$ is a linear space, indeed a reproducing kernel Hilbert space with reproducing kernel $\widehat{K}_{S}(z, w)$ given by

$$
\widehat{K}_{S}(z, w)=\left[\begin{array}{cc}
K_{S}(z, w) & \frac{S(z)-S(\bar{w})}{z-\bar{w}} \\
\frac{\widetilde{S}(z)-\widetilde{S}(\bar{w})}{z-\bar{w}} & K_{\widetilde{S}}(z, w)
\end{array}\right] \quad \text { where } \quad \widetilde{S}(z):=S(\bar{z})^{*} .
$$

(2)\&(3) The space $\mathcal{D}(S)$ is invariant under the transformation $\widehat{R}_{0}$ given by

$$
\widehat{R}_{0}:\left[\begin{array}{l}
f(z) \\
g(z)
\end{array}\right] \mapsto\left[\begin{array}{c}
{[f(z)-f(0)] / z} \\
z g(z)-\widetilde{S}(z) f(0)
\end{array}\right]
$$

with adjoint given by

$$
\left(\widehat{R}_{0}\right)^{*}:\left[\begin{array}{l}
f(z) \\
g(z)
\end{array}\right] \mapsto\left[\begin{array}{c}
z f(z)-S(z) g(0) \\
{[g(z)-g(0)] / z}
\end{array}\right]
$$

Moreover, the following norm identities hold:

$$
\begin{aligned}
\left\|\widehat{R}_{0}\left(\left[\begin{array}{l}
f \\
g
\end{array}\right]\right)\right\|^{2} & =\left\|\left[\begin{array}{l}
f \\
g
\end{array}\right]\right\|_{\mathcal{D}(S)}^{2}-\|f(0)\|_{\mathcal{Y}}^{2}, \\
\left\|\left(\widehat{R}_{0}\right)^{*}\left(\left[\begin{array}{l}
f \\
g
\end{array}\right]\right)\right\|^{2} & =\left\|\left[\begin{array}{l}
f \\
g
\end{array}\right]\right\|_{\mathcal{D}(S)}^{2}-\|g(0)\|_{\mathcal{U}}^{2} .
\end{aligned}
$$

(4) Let $\widehat{\mathbf{U}}_{S}$ be the colligation matrix given by

$$
\widehat{\mathbf{U}}_{S}=\left[\begin{array}{cc}
\widehat{A}_{S} & \widehat{B}_{S} \\
\widehat{C}_{S} & \widehat{D}_{S}
\end{array}\right]:\left[\begin{array}{c}
\mathcal{D}(S) \\
\mathcal{U}
\end{array}\right] \rightarrow\left[\begin{array}{c}
\mathcal{D}(S) \\
\mathcal{Y}
\end{array}\right]
$$

where

$$
\begin{aligned}
& \widehat{A}_{S}:=\left.\widehat{R}_{0}\right|_{\mathcal{D}(S)}, \quad \widehat{B}_{S}: u \mapsto\left[\begin{array}{c}
\frac{S(z)-S(0)}{z} u \\
K_{\widetilde{S}}(z, w) u
\end{array}\right], \\
& \widehat{C}_{S}:\left[\begin{array}{l}
f(z) \\
g(z)
\end{array}\right] \mapsto f(0), \quad \widehat{D}_{S}=S(0) .
\end{aligned}
$$

Then $\widehat{\mathbf{U}}_{S}$ is unitary, and $S$ is recovered as the characteristic function of $\widehat{\mathbf{U}}_{S}$ :

$$
S(z)=\widehat{D}_{S}+z \widehat{C}_{S}\left(I-z \widehat{A}_{S}\right)^{-1} \widehat{B}_{S}
$$

(5) The operator $T$ on a Hilbert space $\mathcal{X}$ is unitarily equivalent to an operator of the form $\widehat{R}_{0}$ on a model space $\mathcal{H}_{S}$ for a contractive 
operator-valued function $S$ on $\mathbb{D}$ if and only if $T$ is a completely non-unitary contraction, i.e., $\|T\| \leq 1$ and

$$
\left(\bigcap_{n \geq 0}\left\{x:\left\|T^{* n} x\right\|=\|x\|\right\}\right) \bigcap\left(\bigcap_{n \geq 0}\left\{x:\left\|T^{n} x\right\|=\|x\|\right\}\right)=\{0\} .
$$

\section{The De Branges-Rovnyak Space $\mathcal{H}(S)$ : other FORMULATIONS}

Later, operator theorists, beginning with Douglas [D1965] and continuing with Fillmore and Williams [FW1971], Sarason [S1986, S1994], Ando [A1990] and Nikolskii-Vasyunin [NV1986, NV1989, NV1998, became interested in giving a more operator-theoretic formulation for de Branges-Rovnyak spaces leading to better insights into the results from the point of view of operator theory; there were also unpublished notes of Rosenblum and Douglas [R1967, D1972]. To carry this out, one needs a generalization of closed subspace of a Hilbert space, namely, contractively included subspace of a Hilbert space, and the notion of the complementary space more general than the familiar notion of the orthogonal complement for an isometrically included closed subspace of a Hilbert space.

3.1. Lifted-norm spaces. Suppose that $\mathcal{M}$ and $\mathcal{H}$ are Hilbert spaces with $\mathcal{M}$ a subset of $\mathcal{H}$ but with its own norm $\|\cdot\|_{\mathcal{M}}$ possibly distinct from the norm it inherits from $\mathcal{H}$ as a subset of $\mathcal{H}$. The terminology - em $\mathcal{M}$ is contractively included in $\mathcal{H}$ shall mean that the inclusion map $\iota: \mathcal{M} \rightarrow \mathcal{H}$ is contractive, i.e.,

$$
\|x\|_{\mathcal{H}}^{2} \leq\|x\|_{\mathcal{M}}^{2} \quad \text { for all } \quad x \in \mathcal{M}
$$

Then one may define an operator $P$ on $\mathcal{H}$ by $P=\iota \iota^{*}$. Then $P=P^{*}$ and

$$
P^{2}=\iota \iota^{*} \iota \iota^{*}=\iota\left(\iota^{*} \iota\right) \iota^{*} \leq \iota \iota^{*}=P, \quad \text { so that } \quad 0 \leq P^{2} \leq P \leq I_{\mathcal{H}} .
$$

Conversely, if $P$ is any positive semidefinite contraction operator $(0 \leq$ $\left.P \leq I_{\mathcal{H}}\right)$, then also $0 \leq P^{2} \leq P \leq I_{\mathcal{H}}$ and one may define a Hilbert space $\mathcal{M}$ as $\mathcal{M}=\operatorname{Ran} P^{\frac{1}{2}}$ with norm given by

$$
\left\|P^{\frac{1}{2}} x\right\|_{\mathcal{M}}=\|\mathbf{Q} x\|_{\mathcal{H}}
$$

(the lifted norm construction) where $\mathbf{Q}$ is the orthogonal projection onto $(\operatorname{Ker} P)^{\perp}=\overline{\operatorname{Ran}} P$. Then one can check that

$$
\left\|P^{\frac{1}{2}} x\right\|_{\mathcal{H}}=\left\|P^{\frac{1}{2}} \mathbf{Q} x\right\|_{\mathcal{H}} \leq\|\mathbf{Q} x\|_{\mathcal{H}}=\left\|P^{\frac{1}{2}} x\right\|_{\mathcal{M}},
$$


so $\mathcal{M}$ is contractively included in $\mathcal{H}$. Moreover, the computation, for $x=P^{\frac{1}{2}} x_{1} \in \mathcal{M}$ and $y \in \mathcal{H}$,

$$
\langle\iota x, y\rangle_{\mathcal{H}}=\left\langle\iota P^{\frac{1}{2}} x_{1}, y\right\rangle_{\mathcal{H}}=\left\langle P^{\frac{1}{2}} x_{1}, y\right\rangle_{\mathcal{H}}=\left\langle P^{\frac{1}{2}} x_{1}, P y\right\rangle_{\mathcal{M}}=\langle x, P y\rangle_{\mathcal{M}}
$$

shows that

$$
\iota^{*}: y \in \mathcal{H} \mapsto P y \in \mathcal{M}
$$

Therefore, $\iota \iota^{*}=P$ as an operator on $\mathcal{H}$. In the sequel the notation $\mathcal{M}=\mathcal{H}_{P}^{l}$ (the lifted-norm space associated with the selfadjoint contraction $P$ ) will be used whenever the space $\mathcal{M}$ contractively included in $\mathcal{H}$ arises in this way from the operator $P \in \mathcal{L}(\mathcal{H})$ with $0 \leq P \leq I_{\mathcal{H}}$. This discussion leads to the following observation.

Proposition 3.1. Contractively included subspaces $\mathcal{M}$ of a Hilbert space $\mathcal{H}$ are in one-to-one correspondence with positive semidefinite contraction operators $P$ on $\mathcal{H}\left(0 \leq P^{2} \leq P \leq I_{\mathcal{H}}\right)$ according to the formula

$$
P=\iota \iota^{*}
$$

where $\iota: \mathcal{M} \rightarrow \mathcal{H}$ is the inclusion map, and then

$$
\mathcal{M}=\operatorname{Ran} P^{\frac{1}{2}} \quad \text { with } \quad\left\|P^{\frac{1}{2}} g\right\|_{\mathcal{M}}=\|\mathbf{Q} g\|_{\mathcal{H}}
$$

where $\mathbf{Q}$ is the orthogonal projection of $\mathcal{H}$ onto $\overline{\operatorname{Ran}} P=(\operatorname{Ker} P)^{\perp}$, written as $\mathcal{M}=\mathcal{H}_{P}^{l}$.

The case where $\mathcal{M}$ is isometrically included in $\mathcal{H}$ corresponds to the case where $P^{2}=P$ and then $P$ is the orthogonal projection of $\mathcal{H}$ onto $\mathcal{M}$.

It is of interest that, even when $P$ is not an orthogonal projection, the lifted-norm space $\mathcal{H}_{I-P}^{l}$ can be viewed as a kind of generalized complementary space (Brangesian complement in the terminology of [S1994]) $\mathcal{M}^{[\perp]}$ to $\mathcal{M}=\mathcal{H}_{P}^{l}$ as explained by the following proposition.

Proposition 3.2. Let $P \in \mathcal{L}(\mathcal{H})$ with $0 \leq P \leq I_{\mathcal{H}}$ and set

$$
\mathcal{M}=\mathcal{H}_{P}^{l}, \quad \mathcal{M}^{[\perp]}=\mathcal{H}_{Q}^{l} \quad \text { where } \quad Q=I-P .
$$

Then $\mathcal{M}$ and $\mathcal{M}^{[\perp]}$ are complementary in the following sense: each $f \in \mathcal{H}$ has a (not necessarily unique) decomposition $f=g+h$ with $g \in \mathcal{M}$ and $h \in \mathcal{M}^{[\perp]}$. Moreover, the norm of $f$ in $\mathcal{H}$ is given by $\|f\|^{2}=\inf \left\{\|g\|_{\mathcal{M}}^{2}+\|h\|_{\mathcal{M}[\perp]}^{2}: g \in \mathcal{M}\right.$ and $h \in \mathcal{M}^{[\perp]}$ such that $\left.f=g+h\right\}$.

\section{Moreover:}

(1) The infimum in (3.1) is attained when $g=P f \in \mathcal{H}_{P}^{l}$ and $h=Q f \in \mathcal{H}_{Q}^{l}$. 
(2) The space $\mathcal{M}^{[\perp]}=\mathcal{H}_{Q}^{l}(Q=I-P)$ can alternatively be characterized as

$$
\mathcal{M}^{[\perp]}=\left\{h \in \mathcal{H}:\|h\|_{Q}^{2}:=\sup \left\{\|g+h\|_{\mathcal{H}}^{2}-\|g\|_{\mathcal{H}_{P}^{l}}^{2}: g \in \mathcal{H}_{P}^{l}\right\}<\infty\right\}
$$

and then $\|h\|_{\mathcal{H}_{Q}^{l}}=\|h\|_{Q}$.

Proof. Note first that, since $P+Q=I_{\mathcal{H}}$ by definition, any $f \in \mathcal{H}$ has a decomposition $f=P f+Q f$ where $g=P f \in \mathcal{H}_{P}^{l}$ and $h=Q f \in \mathcal{H}_{Q}^{l}$.

Next assume that $f=g+h$ with $g \in \mathcal{H}_{P}^{l}$ and $h \in \mathcal{H}_{Q}^{l}$. By Proposition 3.1 one can find $g_{1} \in \overline{\operatorname{Ran}} P$ and $h_{1} \in \overline{\operatorname{Ran}} Q$ so that

$$
g=P^{\frac{1}{2}} g_{1}, \quad h=Q^{\frac{1}{2}} h_{1}, \quad\left(g_{1} \in \overline{\operatorname{Ran}} P\right) .
$$

Taking into account that $P+Q=I_{\mathcal{H}}$ and that $P^{\frac{1}{2}} Q^{\frac{1}{2}}=Q^{\frac{1}{2}} P^{\frac{1}{2}}$, one then computes

$$
\begin{aligned}
& \|g\|_{\mathcal{H}_{P}^{l}}^{2}+\|h\|_{\mathcal{H}_{Q}^{l}}^{2}-\|f\|_{\mathcal{H}}^{2}=\left\|g_{1}\right\|_{\mathcal{H}}^{2}+\left\|h_{1}\right\|_{\mathcal{H}}^{2}-\left\|P^{\frac{1}{2}} g_{1}+Q^{\frac{1}{2}} h_{1}\right\|_{\mathcal{H}}^{2} \\
& \quad=\left\|g_{1}\right\|_{\mathcal{H}}^{2}+\left\|h_{1}\right\|_{\mathcal{H}}^{2}-\left\langle P g_{1}, g_{1}\right\rangle_{\mathcal{H}}-2 \operatorname{Re}\left\langle P^{\frac{1}{2}} g_{1}, Q^{\frac{1}{2}} h_{1}\right\rangle_{\mathcal{H}}-\left\langle Q h_{1}, h_{1}\right\rangle_{\mathcal{H}} \\
& \quad=\left\langle Q g_{1}, g_{1}\right\rangle_{\mathcal{H}}+\left\langle P h_{1}, h_{1}\right\rangle_{\mathcal{H}}-2 \operatorname{Re}\left\langle Q^{\frac{1}{2}} g_{1}, P^{\frac{1}{2}} h_{1}\right\rangle_{\mathcal{H}} \\
& \quad=\left\|Q^{\frac{1}{2}} g_{1}-P^{\frac{1}{2}} h_{1}\right\|_{\mathcal{H}}^{2} \geq 0
\end{aligned}
$$

with equality if and only if $Q^{\frac{1}{2}} g_{1}=P^{\frac{1}{2}} h_{1}$.

To check property (1), note that $g=P f \Rightarrow g_{1}=P^{\frac{1}{2}} f$ and $h=$ $Q f \Rightarrow h_{1}=Q^{\frac{1}{2}} f$. Therefore,

$$
Q^{\frac{1}{2}} g_{1}=Q^{\frac{1}{2}} P^{\frac{1}{2}} f=P^{\frac{1}{2}} Q^{\frac{1}{2}} f=P^{\frac{1}{2}} h_{1}
$$

and hence equality occurs in (3.1) with this choice of $g$ and $h$. Uniqueness follows from the general fact that closed convex sets in a Hilbert space have a unique element of minimal norm.

It remains only to verify statement (2) in the proposition. Given $h \in \mathcal{H}_{Q}^{l}$, define $\|h\|_{Q}$ as in condition (3.2):

$$
\|h\|_{Q}^{2}=\sup _{g \in \mathcal{H}_{P}^{l}}\left\{\|g+h\|_{\mathcal{H}}^{2}-\|g\|_{\mathcal{H}_{P}^{l}}^{2}\right\} .
$$

It has been already shown that, for any $g \in \mathcal{H}_{P}^{l}$,

$$
\|g+h\|_{\mathcal{H}}^{2} \leq\|g\|_{\mathcal{H}_{P}^{l}}^{2}+\|h\|_{\mathcal{H}_{P}^{l}}^{2},
$$

from which it follows that

$$
\|h\|_{\mathcal{H}_{Q}^{l}}^{2} \geq\|h\|_{Q}^{2} .
$$


The following is an alternative direct proof of (3.5) which provides some additional information which will be needed later. Take $h \in \mathcal{H}_{Q}^{l}$ and $g \in \mathcal{H}_{P}^{l}$ in the form (3.3). Computation (3.4) gives

$$
\begin{aligned}
& \|h\|_{\mathcal{H}_{Q}^{l}}^{2}-\|g+h\|_{\mathcal{H}}^{2}+\|g\|_{\mathcal{H}_{P}^{l}}^{2} \\
& \quad=\left\|Q^{\frac{1}{2}} h_{1}\right\|_{\mathcal{H}_{Q}^{l}}^{2}-\left\|P^{\frac{1}{2}} g_{1}+Q^{\frac{1}{2}} h_{1}\right\|_{\mathcal{H}}^{2}+\left\|P^{\frac{1}{2}} g_{1}\right\|_{\mathcal{H}_{P}^{l}}^{2} \\
& \quad=\left\|P^{\frac{1}{2}} h_{1}-Q^{\frac{1}{2}} g_{1}\right\|_{\mathcal{H}}^{2} \geq 0
\end{aligned}
$$

from which (3.5) follows.

Suppose now that $h \in \mathcal{H}$ with $\|h\|_{Q}<\infty$. It suffices to show that $h \in \mathcal{H}_{Q}^{l}=\operatorname{Ran} Q^{\frac{1}{2}}$ and that the reverse inequality

$$
\|h\|_{\mathcal{H}_{Q}^{l}}^{2} \leq\|h\|_{Q}^{2}
$$

holds. From the fact that $\|h\|_{Q}^{2}<\infty$ one can see that

$$
\|h\|_{\mathcal{H}}^{2}+2 \operatorname{Re}\left\langle h, P^{\frac{1}{2}} g_{1}\right\rangle_{\mathcal{H}}+\left\|P^{\frac{1}{2}} g_{1}\right\|^{2} \leq M+\left\|g_{1}\right\|^{2}
$$

for all $g_{1} \in \overline{\operatorname{Ran}} P$ for some constant $M<\infty$. It thus follows that

$$
\begin{aligned}
0 & \leq M-\langle h, h\rangle_{\mathcal{H}}-2 \operatorname{Re}\left\langle h, P^{\frac{1}{2}} g_{1}\right\rangle_{\mathcal{H}}+\left\|g_{1}\right\|^{2}-\left\|P^{\frac{1}{2}} g_{1}\right\|^{2} \\
& =M-\|h\|_{\mathcal{H}}^{2}-2 \operatorname{Re}\left\langle h, P^{\frac{1}{2}} g_{1}\right\rangle_{\mathcal{H}}+\left\langle(I-P) g_{1}, g_{1}\right\rangle_{\mathcal{H}} \\
& =M-\|h\|_{\mathcal{H}}^{2}-2 \operatorname{Re}\left\langle h, P^{\frac{1}{2}} g_{1}\right\rangle_{\mathcal{H}}+\left\|Q^{\frac{1}{2}} g_{1}\right\|_{\mathcal{H}}^{2}
\end{aligned}
$$

for all $g_{1} \in \overline{\operatorname{Ran}} P$. Set

$$
M_{1}:=M-\|h\|_{\mathcal{H}}^{2} .
$$

Then $M_{1} \geq 0$ since one may choose $g_{1}=0$ in the inequality (3.8). Replacing $g_{1}$ by $\omega t g_{1}$ where $t$ is an arbitrary real number and $\omega$ is an appropriate unimodular constant, (3.8) may be rewritten in the form

$$
M_{1}-2\left|\left\langle h, P^{\frac{1}{2}} g_{1}\right\rangle_{\mathcal{H}}\right| t+\left\|Q^{\frac{1}{2}} g_{1}\right\|_{\mathcal{H}}^{2} t^{2} \geq 0 \text { for all real } t .
$$

The Quadratic Formula test for the roots of a real polynomial implies that

$$
\left|\left\langle h, P^{\frac{1}{2}} g_{1}\right\rangle_{\mathcal{H}}\right|^{2} \leq M_{1}\left\|Q^{\frac{1}{2}} g_{1}\right\|^{2} .
$$

The Riesz representation theorem for a linear functional on a Hilbert space then implies that there is an $\widetilde{h} \in \overline{\operatorname{Ran}} Q$ so that

$$
\left\langle h, P^{\frac{1}{2}} g_{1}\right\rangle_{\mathcal{H}}=\left\langle\widetilde{h}, Q^{\frac{1}{2}} g_{1}\right\rangle_{\mathcal{H}} \text {. }
$$

It now follows from this last identity that $P^{\frac{1}{2}} h=Q^{\frac{1}{2}} \widetilde{h}$ and

$$
h=P h+Q h=P^{\frac{1}{2}} Q^{\frac{1}{2}} \widetilde{h}+Q h=Q^{\frac{1}{2}} P^{\frac{1}{2}} \widetilde{h}+Q h=Q^{\frac{1}{2}} h_{1}
$$


with $h_{1}=P^{\frac{1}{2}} \widetilde{h}+Q^{\frac{1}{2}} h$, which implies that $h \in \mathcal{H}_{Q}^{l}$. Furthermore, for $h_{1}$ as defined above,

$$
P^{\frac{1}{2}} h_{1}=P^{\frac{1}{2}}\left(P^{\frac{1}{2}} \widetilde{h}+Q^{\frac{1}{2}} h\right)=P \widetilde{h}+P^{\frac{1}{2}} Q^{\frac{1}{2}} h=(I-Q) \widetilde{h}+Q^{\frac{1}{2}} P^{\frac{1}{2}} h
$$

is in $\overline{\operatorname{Ran}} Q, \widetilde{h}$ itself was arranged to be in $\overline{\operatorname{Ran}} Q$. By Proposition 3.1. any $g \in \mathcal{H}_{P}^{l}$ can be written as $g=P^{\frac{1}{2}} g_{1}$ with $g_{1} \in \overline{\operatorname{Ran}} P$. For this arbitrary $g$ and for $h=Q^{\frac{1}{2}} h_{1} \in \mathcal{H}_{Q}^{l}$ (see (3.6)), it holds that

$$
\|g+h\|_{\mathcal{H}}^{2}-\|g\|_{\mathcal{H}_{P}^{l}}^{2}=\|h\|_{\mathcal{H}_{Q}^{l}}^{2}-\left\|P^{\frac{1}{2}} h_{1}-Q^{\frac{1}{2}} g_{1}\right\|_{\mathcal{H}}^{2}
$$

Since $P^{\frac{1}{2}} h_{1}$ is in $\overline{\operatorname{Ran}} Q$, it follows that

$$
\inf _{g_{1} \in \mathcal{H}}\left\|P^{\frac{1}{2}} h_{1}-Q^{\frac{1}{2}} g_{1}\right\|=0 .
$$

Since $P$ and $Q$ commute, one even has

$$
\inf _{g_{1} \in \overline{\operatorname{Ran}} P}\left\|P^{\frac{1}{2}} h_{1}-Q^{\frac{1}{2}} g_{1}\right\|=0
$$

Combining this with (3.11) and (3.2) leads to the reverse inequality (3.7), and completes the verification of statement (2) in Proposition 3.2 .

Note next that if $\mathcal{M}=\mathcal{H}_{P}^{l}$, then $\mathcal{M}^{[\perp]}=\mathcal{H}_{Q}^{l}$ with $Q=I-P$. Hence for the complementary space $\left(\mathcal{M}^{[\perp]}\right)^{[\perp]}$ of $\mathcal{M}^{[\perp]}$,

$$
\left(\mathcal{M}^{[\perp]}\right)^{[\perp]}=\mathcal{H}_{I-Q}^{l}=\mathcal{H}_{P}^{l}=\mathcal{M}
$$

i.e., one comes back to $\mathcal{M}$ itself. The following corollary is immediate from this observation combined with Proposition 3.2 .

Corollary 3.3. In addition to the complementary space $\mathcal{M}^{[\perp]}$ being recovered from $\mathcal{M}$ via the criterion (3.2), one can also recover $\mathcal{M}=\mathcal{H}_{P}^{l}$ from $\mathcal{M}^{[\perp]}=\mathcal{H}_{Q}^{l}(Q=I-P)$ in the same way:

$$
\mathcal{M}=\left\{g \in \mathcal{H}:\|g\|_{P}^{2}:=\sup _{h \in \mathcal{H}_{Q}^{l}}\|g+h\|_{\mathcal{H}}^{2}-\|h\|_{\mathcal{H}_{Q}^{l}}^{2}<\infty\right\} .
$$

The next Proposition presents the role of the overlapping space in measuring the extent to which the Brangesian complementary space fails to be a true orthogonal complement.

Proposition 3.4. The map $\Xi: f \oplus g \mapsto f+g$ is a partial isometry from $\mathcal{M} \oplus \mathcal{M}^{[\perp]}$ onto $\mathcal{H}$. Furthermore, if one introduces the overlapping space $\mathcal{L}_{P \cdot Q}$ by

$$
\mathcal{L}_{P \cdot Q}=\mathcal{M}^{[\perp]} \cap \mathcal{M}=\mathcal{H}_{P}^{l} \cap \mathcal{H}_{Q}^{l} \quad \text { with norm } \quad\|f\|_{\mathcal{L}_{P \cdot Q}}^{2}=\|f\|_{\mathcal{H}_{P}^{l}}^{2}+\|f\|_{\mathcal{H}_{Q}^{p}}^{2}
$$


then the kernel of the linear transformation $\Xi: \mathcal{M}^{[\perp]} \oplus \mathcal{M} \rightarrow \mathcal{H}$ is given by

$$
\operatorname{Ker} \Xi=\left\{f \oplus-f \in \mathcal{M} \oplus \mathcal{M}^{[\perp]}: f \in \mathcal{L}_{P \cdot Q}\right\}
$$

and the map $\widehat{\Xi}: \mathcal{M}^{[\perp]} \oplus \mathcal{M} \rightarrow \mathcal{H} \oplus \mathcal{L}_{T}$ given by

$$
\widehat{\Xi}: f \oplus g \mapsto(f+g) \oplus k \quad \text { where } \quad k \oplus-k=P_{\text {Ker } \Xi}(f \oplus g)
$$

is unitary.

Proof. This follows essentially from the definitions.

3.2. Pullback spaces. The following variant of the lifted-norm construction given above will be useful in the sequel. Let $T \in \mathcal{L}\left(\mathcal{H}_{0}, \mathcal{H}\right)$ be any contraction operator between two Hilbert spaces $\mathcal{H}_{0}$ and $\mathcal{H}$ (in particular, even if $\mathcal{H}_{0}=\mathcal{H}, T$ is not necessarily positive or even selfadjoint) and set $\mathcal{M}=\operatorname{Ran} T$ with norm given by

$$
\|T x\|_{\mathcal{M}}=\|\mathbf{Q} x\|_{\mathcal{H}}
$$

where $\mathbf{Q}$ is the orthogonal projection of $\mathcal{H}_{0}$ onto $(\operatorname{Ker} T)^{\perp}=\overline{\operatorname{Ran}} T^{*}$ (the pull-back construction). As $T$ is an isometry from the complete space $\operatorname{Ran} \mathbf{Q}$ in the $\mathcal{H}_{0}$-norm onto $\mathcal{M}$, it is easily seen that $\mathcal{M}$ so defined is a Hilbert space. Whenever the Hilbert space $\mathcal{M}$ contractively included in $\mathcal{H}$ has the form $\mathcal{M}=\operatorname{RanT}$ for a contraction operator $T$ with norm given by (3.12), the notation $\mathcal{M}=\mathcal{H}_{T}^{p}$ (the pull-back space associated with $T$ ) shall be applied.

Suppose that $\mathcal{M}=\mathcal{H}_{T}^{p}$ and let $\iota: \mathcal{M}=\operatorname{Ran} T \rightarrow \mathcal{H}$ be the inclusion map. The following computation

$$
\langle\iota T x, y\rangle_{\mathcal{H}}=\langle T x, y\rangle_{\mathcal{H}}=\left\langle x, T^{*} y\right\rangle_{\mathcal{H}}=\left\langle T x, T T^{*} y\right\rangle_{\mathcal{M}}
$$

shows that

$$
\iota^{*}: y \in \mathcal{H} \mapsto T T^{*} y \in \mathcal{M}
$$

and hence $\iota \iota^{*}=T T^{*}=: P$ as an operator on $\mathcal{H}$. Therefore the pullback space $\mathcal{H}_{T}^{p}$ is isometrically equal to the lifted norm space $\mathcal{H}_{T T^{*}}^{l}$, and the lifted-norm space $\mathcal{H}_{P}^{l}$ (where $0 \leq P \leq I$ ) is isometrically equal to the pull-back space $\mathcal{H}_{P^{1 / 2}}^{p}$.

While a lifted norm space uniquely determines the associated positive contraction $P\left(\mathcal{H}_{P}^{l}=\mathcal{H}_{P^{\prime}}^{l} \Leftrightarrow P=P^{\prime}\right)$, pullback spaces determine the associated contraction operator only up to a partially isometric right factor: $\mathcal{H}_{T}^{p}=\mathcal{H}_{T^{\prime}}^{p}$ if and only if there is a partial isometry $\alpha: \mathcal{H}_{0} \rightarrow \mathcal{H}_{0}^{\prime}$ so that $T=T^{\prime} \alpha$ and $T^{\prime}=T \alpha^{*}$.

In conclusion, it follows that all the observations made in the previous section concerning lifted-norm spaces apply equally well to pullback spaces. In general, the Brangesian complementary spaces $\left(\mathcal{H}_{T}^{p}\right)^{[\perp]}$ to 
the pullback space $\mathcal{H}_{T}^{p}$ can be identified with the lifted-norm space $\mathcal{H}_{I-T T^{*}}^{l}$ (or equivalently, the pullback space $\mathcal{H}_{\left(I-T T^{*}\right)^{\frac{1}{2}}}^{p}$. An immediate consequence of these observations and Corollary 3.3 is: given a contraction operator $T \in \mathcal{L}\left(\mathcal{H}_{0}, \mathcal{H}\right)$, the space $\mathcal{H}_{T}^{p}$ can be characterized as

$$
\mathcal{H}_{T}^{p}=\left\{h \in \mathcal{H}: \sup _{g \in \mathcal{H}_{I-T T^{*}}^{l}}\left\{\|g+h\|_{\mathcal{H}}^{2}-\|g\|_{\mathcal{H}_{I-T T^{*}}^{l}}^{2}\right\}<\infty\right\}
$$

(see also [FW1971, Theorem 4.1]). Moreover, the pullback spaces $\mathcal{H}_{T}^{p}$ is isometrically included in $\mathcal{H}$ exactly when $T T^{*}$ is a projection, i.e., when $T$ is a partial isometry.

The overlapping space $\mathcal{L}_{P \cdot Q}$ construction in Proposition 3.4 has a slightly different form in the original de Branges-Rovnyak theory dBR1966a, dBR1966b for the special case where $P=I-T T^{*}$ and $Q=T T^{*}$ which will be now described. In the case where $T$ is an isometry (not just a partial isometry as came up in the previous paragraph), then the operator

$$
\left[\iota_{\mathcal{H}_{I-t T^{*}}^{l}} T\right]: f \oplus g \mapsto f+T g
$$

is unitary from $\mathcal{H}_{I-T T^{*}}^{l} \oplus \mathcal{H}_{0}$ onto $\mathcal{H}$. The de Branges-Rovnyak overlapping space $\mathcal{L}_{T}$ measures the extent to which $\left[\iota_{\mathcal{H}_{I-T T^{*}}^{l}} I_{\mathcal{H}_{0}}\right]$ fails to be isometric for the case of a general contraction operator $T \in \mathcal{L}\left(\mathcal{H}_{0}, \mathcal{H}\right)$. Define the space $\mathcal{L}_{T}$ by

$$
\mathcal{L}_{T}=\left\{f \in \mathcal{H}_{0}: T f \in \mathcal{H}_{I-T T^{*}}^{l}\right\}
$$

with norm given by

$$
\|f\|_{\mathcal{L}_{T}}^{2}=\|T f\|_{\mathcal{H}_{I-T T^{*}}^{l}}^{2}+\|f\|_{\mathcal{H}_{0}}^{2} .
$$

Proposition 3.5. For a contraction operator $T \in \mathcal{L}\left(\mathcal{H}_{0}, \mathcal{H}\right)$ define the overlapping space $\mathcal{L}_{T}$ via (3.13), (3.14). Let $\Xi_{T}: \mathcal{H}_{I-T T^{*}}^{l} \oplus \mathcal{H}_{0} \rightarrow \mathcal{H}$ be the operator given by

$$
\Xi_{T}=\left[\iota_{\mathcal{H}_{I-T T^{*}}^{l}} T\right]:\left[\begin{array}{l}
f \\
g
\end{array}\right] \mapsto f+T g .
$$

Then $\Xi_{T}$ is a coisometry from $\mathcal{H}_{I-T T^{*}}^{l} \oplus \mathcal{H}_{0}$ onto $\mathcal{H}$ with kernel given by

$$
\operatorname{Ker} \Xi_{T}=\left\{\left[\begin{array}{c}
T f \\
-f
\end{array}\right]: f \in \mathcal{L}_{T}\right\}
$$

and the map

$\widehat{\Xi}_{T}:\left[\begin{array}{l}f \\ g\end{array}\right] \mapsto\left[\begin{array}{c}f+T g \\ h\end{array}\right]$ where $h \in \mathcal{L}_{T}$ is determined by $\left[\begin{array}{c}T h \\ -h\end{array}\right]=P_{\mathrm{Ker} \Xi_{T}}\left[\begin{array}{l}f \\ g\end{array}\right]$ 
is unitary from $\mathcal{H}_{I-T T^{*}}^{l} \oplus \mathcal{H}_{0}$ onto $\mathcal{H} \oplus \mathcal{L}_{T}$. Moreover, the overlapping space $\mathcal{L}_{T}$ is itself isometrically equal to a lifted norm space:

$$
\mathcal{L}_{T}=\mathcal{H}_{I-T^{*} T}^{l} .
$$

Proof. By definition $T$ is a coisometry from $\mathcal{H}_{0}$ onto $\mathcal{H}_{T}^{p}=\mathcal{H}_{T T^{*}}^{l}$. By Proposition 3.4, the map $\Xi_{T T^{*}}^{l}=\left[\begin{array}{ll}\iota_{\mathcal{H}_{I-T T^{*}}^{l}} & \iota_{\mathcal{H}_{T T^{*}}^{l}}\end{array}\right]$ is a coisometry from $\mathcal{H}_{I-T T^{*}}^{l} \oplus \mathcal{H}_{T T^{*}}^{l}$ onto $\mathcal{H}$. Note next that the factorization

$$
\Xi_{T}=\Xi_{T T^{*}}^{l} \circ\left[\begin{array}{cc}
I_{\mathcal{H}_{T T^{*}}^{l}} & 0 \\
0 & T
\end{array}\right]
$$

exhibits the map $\Xi_{T}$ as the composition of coisometries (here $T$ is viewed as an element of $\left.\mathcal{L}\left(\mathcal{H}_{0}, \mathcal{H}_{T}^{p}\right)\right)$ and hence $\Xi_{T}$ is a coisometry as asserted.

Proposition 3.4 identifies Ker $\Xi_{T T *}^{l}$ as $\left\{f \oplus-f: f \in \mathcal{L}_{P \cdot Q}\right\}$ (where $P=I-T T^{*}$ and $\left.Q=T T^{*}\right)$. From the factorization (3.16) one can see that $\left[\begin{array}{l}f \\ g\end{array}\right] \in \operatorname{Ker} \Xi_{T}$ if and only if $\left[\begin{array}{c}f \\ T g\end{array}\right] \in \operatorname{Ker} \Xi_{T T^{*}}^{l}$. By Proposition 3.4. this means that $\left[\begin{array}{c}f \\ T g\end{array}\right]$ has the form $\left[\begin{array}{c}f \\ -f\end{array}\right]$ with $f \in \mathcal{L}_{P \cdot Q}=\mathcal{H}_{I-T T^{*}}^{l} \cap \mathcal{H}_{T}^{p}$. Thus $T g=-f \in \mathcal{H}_{I-T T^{*}}^{l} \cap \mathcal{H}_{T}^{p}$ so $g \in \mathcal{L}_{T}$ and $\left[\begin{array}{c}-T g \\ g\end{array}\right] \in \operatorname{Ker} \Xi_{T}$. The unitary property of $\widehat{\Xi}_{T}$ now follows easily.

It remains only to verify that $\mathcal{L}_{T}=\mathcal{H}_{I-T^{*} T}^{l}$ isometrically. Suppose first that $g=\left(I-T^{*} T\right)^{\frac{1}{2}} g_{1} \in \mathcal{H}_{I-T^{*} T}^{l}$. Then certainly $g \in \mathcal{H}_{0}$. But also,

$$
T g=T\left(I-T^{*} T\right)^{\frac{1}{2}} g_{1}=\left(I-T T^{*}\right)^{\frac{1}{2}} T g_{1} \in \mathcal{H}_{I-T T^{*}}^{l} .
$$

Moreover, the same intertwining $T\left(I-T^{*} T\right)=\left(I-T T^{*}\right) T$ implies that $T g_{1} \in \overline{\operatorname{Ran}} \mathcal{H}_{I-T T^{*}}^{l}$ since $g_{1} \in \overline{\operatorname{Ran}}\left(I-T^{*} T\right)$. Therefore,

$$
\begin{aligned}
\|g\|_{\mathcal{L}_{T}}^{2} & =\|h\|_{\mathcal{H}_{0}}^{2}+\|T g\|_{\mathcal{H}_{I-T T^{*}}^{l}}^{2} \\
& =\left\|\left(I-T^{*} T\right)^{\frac{1}{2}} g_{1}\right\|_{\mathcal{H}_{0}}^{2}+\left\|\left(I-T T^{*}\right)^{\frac{1}{2}} T g_{1}\right\|_{\mathcal{H}_{I-T T^{*}}^{l}}^{2} \\
& =\left\langle\left(I-T^{*} T\right) g_{1}, g_{1}\right\rangle_{\mathcal{H}_{0}}+\left\|T g_{1}\right\|_{\mathcal{H}}^{2}=\left\|g_{1}\right\|_{\mathcal{H}_{0}}^{2}=\|g\|_{\mathcal{H}_{I-T^{*} T}^{l}}^{2}
\end{aligned}
$$

and the equality of norms follows. Conversely, if $g \in \mathcal{L}_{T}$, then it follows that $g \in \mathcal{H}_{0}$ with $T g \in \mathcal{H}_{I-T^{*} T}^{l}$. Hence there is a $\widetilde{g} \in \overline{\operatorname{Ran}}\left(I-T T^{*}\right)$ so that $T g=\left(I-T T^{*}\right)^{\frac{1}{2}} \widetilde{g}$. Therefore,

$$
\begin{aligned}
g & =T^{*} T g+\left(I-T^{*} T\right) g \\
& =T^{*}\left(I-T T^{*}\right)^{\frac{1}{2}} \widetilde{g}+\left(I-T^{*} T\right) g \\
& =\left(I-T^{*} T\right)^{\frac{1}{2}} T^{*} \widetilde{g}+\left(I-T^{*} T\right) g \in \operatorname{Ran}\left(I-T^{*} T\right)^{\frac{1}{2}}
\end{aligned}
$$


which allows to conclude that $g \in \mathcal{H}_{I-T^{*} T}^{l}$. The isometric equality (3.15) has now been verified.

3.3. Spaces associated with Toeplitz operators. From now on it will be assumed that all Hilbert spaces are separable. For $\mathcal{U}$ a coefficient Hilbert space, let $L^{2}(\mathcal{U})$ denote the Hilbert space of weakly measurable norm-square integrable functions on the unit circle $\mathbb{T}$; in terms of Fourier series representation, one can write

$L^{2}(\mathcal{U})=\left\{f(\zeta) \sim \sum_{n=-\infty}^{\infty} f_{n} \zeta^{n}: f_{n} \in \mathcal{U}\right.$ with $\left.\|f\|_{L^{2}(\mathcal{U})}^{2}:=\sum_{n=-\infty}^{\infty}\left\|f_{n}\right\|^{2}<\infty\right\}$.

The vector-valued Hardy space $H^{2}(\mathcal{U})$ is the subspace of $L^{2}(\mathcal{U})$ consisting of functions $f(\zeta)=\sum_{n=0}^{\infty} f_{n} \zeta^{n}$ having $f_{n}=0$ for $n<0$ and can also be viewed as the space of $\mathcal{U}$-valued analytic functions on the unit disk $\mathbb{D}$ having $L^{2}$-norm along circles of radius $r$ uniformly bounded as $r \uparrow 1$. Given two coefficient Hilbert spaces $\mathcal{U}$ and $\mathcal{Y}$, let $L^{\infty}(\mathcal{U}, \mathcal{Y})$ denote the space of weakly measurable essentially bounded $\mathcal{L}(\mathcal{U}, \mathcal{Y})$ valued functions on $\mathbb{T}(W: \mathbb{T} \rightarrow \mathcal{L}(\mathcal{U}, \mathcal{Y}))$. Given $W \in L^{\infty}(\mathcal{U}, \mathcal{Y})$, let $L_{W}: L^{2}(\mathcal{U}) \rightarrow L^{2}(\mathcal{Y})$ denote the Laurent operator of multiplication by $W$ on vector-valued $L^{2}$ :

$$
L_{W}: f(\zeta) \mapsto W(\zeta) f(\zeta) .
$$

The Toeplitz operator $T_{W}$ associated with $W$ is the compression of $L_{W}$ to the Hardy space:

$$
T_{W}: f \mapsto P_{H^{2}(\mathcal{Y})}\left(L_{W} f\right) \quad \text { for } \quad f \in H^{2}(\mathcal{U}) .
$$

Let $H^{\infty}(\mathcal{U}, \mathcal{Y})$ denote the subspace of $L^{\infty}(\mathcal{U}, \mathcal{Y})$ consisting of $W$ with negative Fourier coefficients vanishing: $W(\zeta) \sim \sum_{n=0}^{\infty} W_{n} \zeta^{n}$; as in the vector-valued case, $W(\zeta)$ can be viewed as the almost everywhere existing nontangential weak-limit boundary value function of an operatorvalued function $z \mapsto W(z)$ on the unit disk $\mathbb{D}$ (here the separability assumption on the coefficient Hilbert spaces is invoked-see e.g. [RR1985] for details). For the case of $W \in H^{\infty}(\mathcal{U}, \mathcal{Y})$, the Toeplitz operator $T_{W}$ assumes the simpler form

$$
T_{W}: f(\zeta) \mapsto W(\zeta) \cdot f(\zeta)
$$

In this case one says that $W$ is an analytic Toeplitz operator (see [RR1985]).

The de Branges-Rovnyak spaces discussed in [BR1966a, Appendix] and dBR1966b] amount to the special case of the constructions in Section 3.2 above applied to the case where $\mathcal{H}_{0}=H^{2}(\mathcal{U}), \mathcal{H}=H^{2}(\mathcal{Y})$ and $T$ is the analytic Toeplitz operator $T=T_{S}$. 
An easy consequence of the characterization of uniqueness discussed above for pullback spaces is the following: two Schur-class functions $S \in \mathcal{S}(\mathcal{U}, \mathcal{Y})$ and $S \in \mathcal{S}\left(\mathcal{U}^{\prime}, \mathcal{Y}\right)$ determine the same pullback space $\mathcal{M}(S)=\mathcal{H}_{T_{S}}^{p}=\mathcal{H}_{T_{S^{\prime}}}^{p}$ (and hence also the same de Branges-Rovnyak space $\mathcal{H}(S)=\mathcal{H}\left(S^{\prime}\right)$ ) if and only if there is a partially isometric multiplier $\alpha$ so that $S=S^{\prime} \alpha$ and $S^{\prime}=S \alpha^{*}$. In particular, there is a choice of Beurling-Lax representer $S$ for a given $\mathcal{M}=\mathcal{H}_{T_{S}}^{p}$ with the additional property that

$$
\{u \in \mathcal{U}: S(z) u \equiv 0\}=\{0\} .
$$

In detail, the following identification of the de Branges-Rovnyak space $\mathcal{H}(S)$ as a lifted-norm space holds.

Proposition 3.6. For an $S \in \mathcal{S}(\mathcal{U}, \mathcal{Y})$, let $\mathcal{H}(S)$ be the de BrangesRovnyak space as defined by (2.1) above. Then $\mathcal{H}(S)$ is isometrically equal to the lifted norm space

$$
\mathcal{H}(S)=\mathcal{H}_{I-T_{S} T_{S}^{*}}^{l}
$$

Equivalently, if $\mathcal{M}(S)$ denotes the pullback space

$$
\mathcal{M}(S):=\mathcal{H}_{T_{S}}^{p},
$$

then $\mathcal{H}(S)$ is the Brangesian complementary space $(\mathcal{M}(S))^{[\perp]}$ to $\mathcal{M}(S)$ in $H^{2}(\mathcal{Y})$.

Proof. All this can be seen from the definition of the $\mathcal{H}(S)$ norm in (2.1) combined with Proposition 3.2 and the equivalence between pullback spaces and lifted norm spaces as explained in Section 3.2 .

The next result indicates how one can get parts (2) and (3) in Theorem 2.1 using the lifted-norm characterization of $\mathcal{H}(S)$. The key tool for this task is the following fundamental result of Douglas.

Proposition 3.7. (See [D1965.) Given two Hilbert space operators $A \in \mathcal{L}\left(\mathcal{H}_{1}, \mathcal{H}_{2}\right)$ and $B \in \mathcal{L}\left(\mathcal{H}_{0}, \mathcal{H}_{2}\right)$, there exists a contraction operator $X \in \mathcal{L}\left(\mathcal{H}_{0}, \mathcal{H}_{1}\right)$ with $A X=B$ if and only if the operator inequality $B B^{*} \leq A A^{*}$ holds.

For $\mathcal{X}$ any coefficient Hilbert space, let $\mathbf{S}_{\mathcal{X}}$ denote the unilateral shift operator of multiplicity $\operatorname{dim} \mathcal{X}$ as modeled on $H^{2}(\mathcal{X})$ :

$$
\mathbf{S}_{\mathcal{X}}: f(z) \mapsto z f(z) \text { for } f \in H^{2}(\mathcal{X}) .
$$

Then it is easily verified that its adjoint is given by the differencequotient transformation:

$$
\mathbf{S}_{\mathcal{X}}^{*}: f(z) \mapsto \frac{f(z)-f(0)}{z} \quad \text { for } \quad f \in H^{2}(\mathcal{X}) .
$$


Now part (2) (apart from an analysis of when equality holds in(2.3) which will come later) and part (3) of Theorem 2.1 can be verified as follows.

Theorem 3.8. Let $S$ be a Schur-class operator-valued function in $\mathcal{S}(\mathcal{U}, \mathcal{Y})$. Then:

(2) The space $\mathcal{H}(S)$ is invariant under the difference-quotient transformation $\mathbf{S}_{\mathcal{Y}}^{*}$ with

$$
\left\|\mathbf{S}_{\mathcal{Y}}^{*} f\right\|_{\mathcal{H}(S)}^{2} \leq\|f\|_{\mathcal{H}(S)}^{2}-\|f(0)\|_{\mathcal{H}(S)}^{2}
$$

(3) For any vector $u \in \mathcal{U}$, the function $\frac{S(z)-S(0)}{z} u$ belongs to $\mathcal{H}(S)$. Let $R_{0}: \mathcal{H}(S) \rightarrow \mathcal{H}(S)$ and $\tau: \mathcal{U} \rightarrow \mathcal{H}(S)$ be the operators $R_{0}=\left.\mathbf{S}_{\mathcal{Y}}^{*}\right|_{\mathcal{H}(S)}$ and $\tau: u \mapsto \frac{S(z)-S(0)}{z} u$. Then $R_{0}^{*} \in \mathcal{L}(\mathcal{H}(S))$ is given by

$$
R_{0}^{*}: f(z) \mapsto z f(z)-S(z) \cdot \tau^{*}(f)
$$

with the norm of $\tau^{*}(f)$ given by

$$
\left\|\tau^{*}(f)\right\|_{\mathcal{U}}^{2}=\|f\|_{\mathcal{H}(S)}^{2}-\left\|R_{0}^{*} f\right\|_{\mathcal{H}(S)} .
$$

The following proof synthesizes arguments from [NV1986] and [S1986]. Proof of (2). View $\mathcal{H}(S)$ as $\operatorname{Ran}\left(I-T_{S} T_{S}^{*}\right)^{\frac{1}{2}}$ and introduce the notation

$$
P_{0}=I-\mathbf{S}_{\mathcal{Y}} \mathbf{S}_{\mathcal{Y}}^{*}
$$

for the projection onto the constant functions in $H^{2}(\mathcal{Y})$. Next observe the identity

$$
\begin{aligned}
& {\left[\begin{array}{ll}
\mathbf{S}_{\mathcal{Y}}\left(I-T_{S} T_{S}^{*}\right)^{\frac{1}{2}} & P_{0}
\end{array}\right]\left[\begin{array}{c}
\left(I-T_{S} T_{S}^{*}\right)^{\frac{1}{2}} S^{*} \\
P_{0}
\end{array}\right]} \\
& \quad=\mathbf{S}_{\mathcal{Y}}\left(I-T_{S} T_{S}^{*}\right) \mathbf{S}_{\mathcal{Y}}^{*}+I-\mathbf{S}_{\mathcal{Y}} \mathbf{S}_{\mathcal{Y}}^{*}=I-T_{S} T_{S}^{*} .
\end{aligned}
$$

Then by the Douglas criterion (Proposition 3.7), there is a contraction operator $\left[\begin{array}{c}X \\ Y\end{array}\right]$ so that

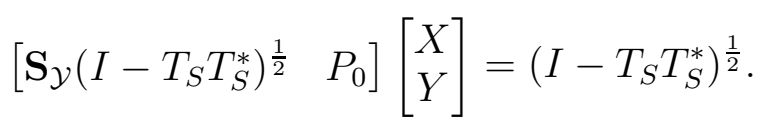

Multiplying on the left by $\mathbf{S}_{\mathcal{Y}}^{*}$ and then by $P_{0}$ successively breaks this up into the pair of equations

$$
\left(I-T_{S} T_{S}^{*}\right)^{\frac{1}{2}} X=\mathbf{S}_{\mathcal{Y}}^{*}\left(I-T_{S} T_{S}^{*}\right)^{\frac{1}{2}}, \quad P_{0} Y=P_{0}\left(I-T_{S} T_{S}^{*}\right)^{\frac{1}{2}}
$$

The first equation in (3.22) reveals that $\mathcal{H}(S)$ is invariant under $\mathbf{S}_{\mathcal{Y}}^{*}$ and

$$
\left\|\mathbf{S}_{\mathcal{Y}}^{*} f\right\|_{\mathcal{H}(S)}^{2}=\left\|X f_{1}\right\|_{H^{2}(\mathcal{Y})}^{2}
$$


(assuming that it is arranged that $\operatorname{Ran} X \subset \overline{\operatorname{Ran}}\left(I-T_{S} T_{S}^{*}\right)$ which is always possible). Moreover, the fact that $\left[\begin{array}{l}X \\ Y\end{array}\right]$ is a contraction implies that $Y$ has the form $Y=K\left(I-X^{*} X\right)^{\frac{1}{2}}$ with $K$ a contraction, and hence

$$
P_{0} K\left(I-X^{*} X\right)^{\frac{1}{2}}=P_{0}\left(I-T_{S} T_{S}^{*}\right)^{\frac{1}{2}}
$$

Then from the second equation in (3.22) one gets

$$
\begin{aligned}
\|f(0)\|_{\mathcal{Y}}^{2} & =\left\|P_{0}\left(I-T_{S} T_{S}^{*}\right)^{\frac{1}{2}} f_{1}\right\|_{\mathcal{Y}}^{2} \\
& \leq\left\|\left(I-X^{*} X\right)^{\frac{1}{2}} f_{1}\right\|_{H^{2}(\mathcal{Y})}^{2} \\
& =\left\|f_{1}\right\|_{H^{2}(\mathcal{Y})}^{2}-\left\|X f_{1}\right\|_{H^{2}(\mathcal{Y})}^{2}=\|f\|_{\mathcal{H}(S)}^{2}-\left\|\mathbf{S}_{\mathcal{Y}}^{*} f\right\|_{\mathcal{H}(S)}^{2},
\end{aligned}
$$

and the norm estimate (3.19) follows.

Proof of (3). Note that the subspace $\left\{\frac{S(z)-S(0)}{z} u: u \in \mathcal{U}\right\}$ is the range of the commutator operator $\mathbf{S}_{\mathcal{Y}}^{*} T_{S}-T_{S} \mathbf{S}_{\mathcal{Y}}^{*}$. Hence to show that $\frac{S(z)-S(0)}{z} u \in$ $\mathcal{H}(S)$ for each $u \in \mathcal{U}$, it suffices to show that $\operatorname{Ran}\left(I-T_{S} T_{S}^{*}\right)^{\frac{1}{2}}$ is invariant under the commutator $\mathbf{S}_{\mathcal{Y}}^{*} T_{S}-T_{S} \mathbf{S}_{\mathcal{Y}}^{*}$. Again by Proposition 3.7, it suffices to show that

$$
\left(\mathbf{S}_{\mathcal{Y}}^{*} T_{S}-T_{S} \mathbf{S}_{\mathcal{Y}}^{*}\right)\left(\mathbf{S}_{\mathcal{Y}}^{*} T_{S}-T_{S} \mathbf{S}_{\mathcal{Y}}^{*}\right)^{*} \leq I-T_{S} T_{S}^{*}
$$

It is readily seen that the left hand side of (3.23) is equal to $\mathbf{S}_{\mathcal{Y}}^{*} T_{S}^{*} T_{S}^{*} \mathbf{S}_{\mathcal{Y}}-$ $T_{S} T_{S}^{*}$, so (3.23) does hold.

Finally, the formula for $R_{0}^{*}$ can be verified as follows. Assume first that $h \in \mathcal{H}(S)$ has the special form $h=\left(I-T_{S} T_{S}^{*}\right) h_{1}$ for some $h_{1} \in$ $H^{2}(\mathcal{Y})$. Then the computation

$$
\begin{aligned}
\left\langle R_{0} g, h\right\rangle_{\mathcal{H}(S)} & =\left\langle\mathbf{S}_{\mathcal{Y}}^{*} g, h\right\rangle_{\mathcal{H}(S)}=\left\langle\mathbf{S}_{\mathcal{Y}}^{*} g, h_{1}\right\rangle_{H^{2}(\mathcal{Y})}=\left\langle g, \mathbf{S}_{\mathcal{Y}} h_{1}\right\rangle_{H^{2}(\mathcal{Y})} \\
& =\left\langle g,\left(I-T_{S} T_{S}^{*}\right) \mathbf{S}_{\mathcal{Y}} h_{1}\right\rangle_{\mathcal{H}(S)}
\end{aligned}
$$

shows that

$$
R_{0}^{*} h=\left(I-T_{S} T_{S}^{*}\right) \mathbf{S}_{\mathcal{Y}} h_{1}=\mathbf{S}_{\mathcal{Y}} h-T_{S}\left(T_{S}^{*} \mathbf{S}_{\mathcal{Y}}-\mathbf{S}_{\mathcal{Y}} T_{S}^{*}\right) h_{1} .
$$

It is easily verified that

$$
\mathbf{S}_{\mathcal{Y}}^{*} T_{S}-T_{S} \mathbf{S}_{\mathcal{Y}}^{*}: h_{1}(z) \rightarrow \frac{S(z)-S(0)}{z} h_{1}(0)=\tau\left(h_{1}(0)\right)
$$

and hence the adjoint action must have the form

$$
\left(T_{S}^{*} \mathbf{S}_{\mathcal{Y}}-\mathbf{S}_{\mathcal{Y}} T_{S}^{*}\right) h_{1}=\widetilde{h}_{1}(0)
$$

where the constant $\widetilde{h}_{1}(0) \in \mathcal{U}$ is determined by

$$
\begin{aligned}
\left\langle\widetilde{h}_{1}(0), u\right\rangle_{\mathcal{U}} & =\left\langle h_{1}, \tau(u)\right\rangle_{H^{2}(\mathcal{Y})}=\left\langle\left(I-T_{S} T_{S}^{*}\right) h_{1}, \tau(u)\right\rangle_{\mathcal{H}(S)}=\langle h, \tau(u)\rangle_{\mathcal{H}(S)} \\
& =\left\langle\tau^{*}(h), u\right\rangle_{\mathcal{U}}
\end{aligned}
$$


where the adjoint is with respect to the $\mathcal{H}(S)$ inner product on the range of $\tau$. Therefore, $\widetilde{h}_{1}(0)=\tau^{*}(h)$ and the formula (3.20) for $R_{0}^{*}$ is now an immediate consequence of (3.24) for the case where $f$ has the special form $f=\left(I-T_{S} T_{S}^{*}\right) f_{1}$. But elements in $\mathcal{H}(S)$ of this special form are dense in $\mathcal{H}(S)$ so the general case of (3.20) now follows by taking limits.

The next task is the computation of the action of $I-R_{0} R_{0}^{*}$ on a general element $f$ of $\mathcal{H}(S)$ :

$$
\begin{aligned}
\left(I-R_{0} R_{0}^{*}\right) f & =f(z)-\frac{1}{z}\left[z f(z)-S(z) \tau^{*}(f)+S(0) \tau^{*}(f)\right] \\
& =\frac{S(z)-S(0)}{z} \tau^{*}(f)=\tau \tau^{*}(f) .
\end{aligned}
$$

Therefore,

$\|f\|_{\mathcal{H}(S)}^{2}-\left\|R_{0}^{*} f\right\|_{\mathcal{H}(S)}^{2}=\left\langle\left(I-R_{0} R_{0}^{*}\right) f, f\right\rangle_{\mathcal{H}(S)}=\left\langle\tau \tau^{*}(f), f\right\rangle_{\mathcal{H}(S)}=\left\|\tau^{*}(f)\right\|_{\mathcal{U}}^{2}$

and the identity (3.21) follows.

The next goal is to show that the estimate (3.19) is enough to verify one direction of part (4) in Theorem 2.1.

Theorem 3.9. Let $S$ be in $\mathcal{S}(\mathcal{U}, \mathcal{Y})$ with associated de Branges-Rovnyak space $\mathcal{H}(S)$ and model operator $R_{0}:=\left.\mathbf{S}_{\mathcal{Y}}^{*}\right|_{\mathcal{H}(S)}$. Then $R_{0}$ is completely non-isometric, i.e., if $f \in \mathcal{H}(S)$ is such that $\left\|R_{0}^{n} f\right\|_{\mathcal{H}(S)}=\|f\|_{\mathcal{H}(S)}$ for $n=0,1,2, \ldots$, then $f=0$.

Proof. Suppose that $f(z)=\sum_{n=0}^{\infty} f_{n} z^{n}$ is in $\mathcal{H}(S)$ with $\left\|R_{0}^{n} f\right\|=\|f\|$ for all $n=1,2,3, \ldots$ Then in particular, from the observation that $f_{n}=\left(R_{0}^{n} f\right)(0)$ together with (3.19) $)$ one gets

$$
\left\|f_{n}\right\|^{2}=\left\|\left(R_{0}^{n} f\right)(0)\right\|_{\mathcal{Y}}^{2} \leq\left\|R_{0}^{n} f\right\|_{\mathcal{H}(S)}^{2}-\left\|R_{0}^{n+1} f\right\|_{\mathcal{H}(S)}^{2}=0
$$

and hence $f_{n}=0$ for all $n=0,1,2, \ldots$, i.e., $f=0$.

3.4. Reproducing kernel Hilbert spaces. A reproducing kernel Hilbert space (RKHS) by definition is a Hilbert space whose elements are functions on some set $\Omega$ with values in a coefficient Hilbert space, say $\mathcal{Y}$, such that the evaluation map $\mathbf{e}(\omega): f \mapsto f(\omega)$ is continuous from $\mathcal{H}$ into $\mathcal{Y}$ for each $\omega \in \Omega$. Associated with any such space is a positive $\mathcal{L}(\mathcal{Y})$-valued kernel on $\Omega$, i.e., a function $K: \Omega \times \Omega \rightarrow \mathcal{L}(\mathcal{Y})$ with the positive-kernel property

$$
\sum_{i, j=1}^{N}\left\langle K\left(\omega_{i}, \omega_{j}\right) y_{j}, y_{i}\right\rangle_{\mathcal{Y}} \geq 0
$$


for any choice of finitely many points $\omega_{1}, \ldots, \omega_{N} \in \Omega$ and vectors $y_{1}, \ldots, y_{N} \in \mathcal{Y}$, which "reproduces" the values of the functions in $\mathcal{H}$ in the sense that

(i) the function $\omega \mapsto K(\omega, \zeta) y$ is in $\mathcal{H}$ for each $\zeta \in \Omega$ and $y \in \mathcal{Y}$, and

(ii) the reproducing formula

$$
\langle f, K(\cdot, \zeta) y\rangle_{\mathcal{H}}=\langle f(\zeta), y\rangle_{\mathcal{Y}}
$$

holds for all $f \in \mathcal{H}, \zeta \in \Omega$, and $y \in \mathcal{Y}$.

Such kernels are also characterized by the Kolmogorov factorization property: there exists a function $H: \Omega \rightarrow \mathcal{L}(\widetilde{\mathcal{H}}, \mathcal{Y})$ for some auxiliary Hilbert spaces $\widetilde{\mathcal{H}}$ so that

$$
K(\omega, \zeta)=H(\omega) H(\zeta)^{*}
$$

One particular way to produce this factorization is by taking $\widetilde{\mathcal{H}}=\mathcal{H}$ and setting $H(\omega)=\mathbf{e}(\omega)$ where $\mathbf{e}(\omega)$ is the point-evaluation map described above. Whenever a Hilbert space of functions arises in this way from a positive kernel $K$, one writes $\mathcal{H}=\mathcal{H}(K)$. An early thorough treatment of RKHSs (for the case $\mathcal{Y}=\mathbb{C}$ ) is the paper of Aronszajn Ar1950; a good recent treatment is in the book of Agler-McCarthy AMcC2002] (where they are called Hilbert function spaces) while the recent papers [BV2003] formulate more general settings (formal commuting or noncommuting variables).

Given a pair of reproducing kernel Hilbert spaces $\mathcal{H}\left(K_{0}\right)$ and $\mathcal{H}(K)$ where say $\mathcal{H}\left(K_{0}\right)$ consists of functions with values in $\mathcal{Y}_{0}$ and $\mathcal{H}(K)$ consists of functions with values in $\mathcal{Y}$, an object of much interest for operator theorists is the space of multipliers $\mathcal{M}\left(K_{0}, K\right)$ consisting of $\mathcal{L}\left(\mathcal{Y}_{0}, \mathcal{Y}\right)$-valued functions $F$ on $\Omega$ with the property that the multiplication operator

$$
M_{F}: f(\omega) \mapsto F(\omega) f(\omega)
$$

maps $\mathcal{H}\left(K_{0}\right)$ into $\mathcal{H}(K)$. The simple computation

$$
\begin{aligned}
\left\langle M_{F} f, K(\cdot, \zeta) y\right\rangle_{\mathcal{H}(K)} & =\langle F(\zeta) f(\zeta), y\rangle_{\mathcal{Y}} \\
& =\left\langle f(\zeta), F(\zeta)^{*} y\right\rangle_{\mathcal{Y}_{0}}=\left\langle f, K_{0}(\cdot, \zeta) F(\zeta)^{*} y\right\rangle_{\mathcal{H}\left(K_{0}\right)}
\end{aligned}
$$

shows that

$$
\left(M_{F}\right)^{*}: K(\cdot, \zeta) y \mapsto K_{0}(\cdot, \zeta) F(\zeta)^{*} y
$$

Therefore

$$
\left\langle\left(I-M_{F} M_{F}^{*}\right) K(\cdot, \zeta) y, K(\cdot, \omega) y^{\prime}\right\rangle_{\mathcal{H}(K)}=\left\langle\left(K(\omega, \zeta)-F(z) K_{0}(\omega, \zeta) F(\zeta)^{*}\right) y, y^{\prime}\right\rangle_{\mathcal{Y}}
$$

so that $F$ is a contractive multiplier from $\mathcal{H}\left(K_{0}\right)$ to $\mathcal{H}(K)$ if and only if the kernel $K(\omega, \zeta)-F(z) K_{0}(\omega, \zeta) F(\zeta)^{*}$ is positive on $\Omega \times \Omega$. By 
letting $K_{0}(\omega, \zeta) \equiv I_{\mathcal{Y}}$ and rescaling, one can arrive at the following proposition [BeB1984].

Proposition 3.10. A function $F: \Omega \rightarrow \mathcal{Y}$ belongs to $\mathcal{H}(K)$ with $\|F\|_{\mathcal{H}(K)} \leq \gamma$ if and only if the kernel $K(\omega, \zeta)-\gamma^{-2} F(z) F(\zeta)^{*}$ is positive on $\Omega \times \Omega$.

A first example of a reproducing kernel Hilbert space is the space $H^{2}(\mathcal{Y})$ (considered as consisting of analytic functions on the unit disk $\mathbb{D})$ with the Szegö kernel tensored with the identity operator on $\mathcal{Y}$ : $k_{\mathrm{Sz}}(z, w) I_{\mathcal{Y}}$ where $k_{\mathrm{Sz}}(z, w)=\frac{1}{1-z \bar{w}}$. The space of multipliers $\mathcal{M}\left(k_{\mathrm{Sz}} I_{\mathcal{U}}, k_{\mathrm{Sz}} I_{\mathcal{Y}}\right)$ between two Hardy spaces can be identified with the space $H^{\infty}(\mathcal{U}, \mathcal{Y})$ of bounded analytic functions on $\mathbb{D}$ with values in $\mathcal{L}(\mathcal{U}, \mathcal{Y})$. Given $F \in H^{\infty}(\mathcal{U}, \mathcal{Y})$, the associated multiplication operator $M_{F}$ is simply the Toeplitz operator $T_{F}$ which was discussed above. Note that in general $F^{*}$ is not a multiplier when $F$ is a multiplier; however it does hold that $M_{F}^{*}=\left(T_{F}\right)^{*}=T_{F^{*}}$ for $F$ a multiplier between two Szegö-kernel RKHSs (i.e., Hardy spaces).

The next task is the identification of the de Branges-Rovnyak space $\mathcal{H}(S)$ (where $S$ is a Schur-class function) as a reproducing kernel Hilbert space in its own right. This fills in part (1) of Theorem 2.1.

Theorem 3.11. The de Branges-Rovnyak space $\mathcal{H}(S)=\mathcal{H}_{T_{S}}^{p}$ associated with a Schur-class function $S \in \mathcal{S}(\mathcal{U}, \mathcal{Y})$ as above is isometrically equal to the reproducing kernel Hilbert space $\mathcal{H}\left(K_{S}\right)$ where $K_{S}$ is the de Branges-Rovnyak kernel

$$
K_{S}(z, w)=\frac{I-S(z) S(w)^{*}}{1-z \bar{w}} .
$$

Proof. As a result of the general identity (3.27), it follows that

$$
T_{S}^{*}: k_{\mathrm{Sz}}(\cdot, w) y \mapsto k_{\mathrm{Sz}}(\cdot, w) S(w)^{*} y
$$

and hence

$$
K_{S}(\cdot, w) y=\left(I-T_{S} T_{S}^{*}\right)\left(k_{\mathrm{Sz}}(\cdot, w) y\right) .
$$

It then follows that $K_{S}(\cdot, w) y \in \mathcal{H}(S)$ for each $w \in \mathbb{D}$ and $y \in \mathcal{Y}$, and also, for $f=\left(I-T_{S} T_{S}^{*}\right) f_{1} \in \mathcal{H}(S)$

$$
\begin{aligned}
\left\langle f, K_{S}(\cdot, w) y\right\rangle_{\mathcal{H}(S)} & =\left\langle f,\left(I-T_{S} T_{S}^{*}\right)\left(k_{\mathrm{Sz}}(\cdot, w) y\right\rangle_{\mathcal{H}(S)}\right. \\
& =\left\langle f, k_{\mathrm{Sz}}(\cdot, w) y\right\rangle_{H^{2}(\mathcal{Y})}=\langle f(w), y\rangle_{\mathcal{Y}}
\end{aligned}
$$

from which one can see that $K_{S}(z, w)$ has all the properties required to be the reproducing kernel for $\mathcal{H}(S)$. 
If $\mathbf{e}(w): \mathcal{H}(S) \rightarrow \mathcal{Y}$ is the evaluation-at-w map

$$
\mathbf{e}(w): f \mapsto f(w),
$$

on the space $\mathcal{H}(S)$, then its adjoint is given by the kernel function for the point $w$ :

$$
\mathbf{e}(w)^{*}: y \mapsto K_{S}(z, w) y=\frac{I-S(z) S(w)^{*}}{1-z \bar{w}}
$$

In particular,

$$
\mathbf{e}(0)^{*}: y \mapsto\left(I-S(z) S(0)^{*}\right) y .
$$

This is the last piece needed to complete the proof of part (4) of Theorem 2.1. Along the way, here also is a completion of the analysis of when the inequality (2.3) holds with equality.

Theorem 3.12. The colligation matrix $\mathbf{U}_{S}=\left[\begin{array}{cc}A_{S} & B_{S} \\ C_{S} & D_{S}\end{array}\right]:\left[\begin{array}{c}\mathcal{H}(S) \\ \mathcal{U}\end{array}\right] \rightarrow$ $\left[\begin{array}{c}\mathcal{H}(S) \\ \mathcal{Y}\end{array}\right]$ given by (2.7) is coisometric and has characteristic function equal to $S$ :

$$
S(z)=D_{S}+z C_{S}\left(I-z A_{S}\right)^{-1} B_{S}
$$

Furthermore, the reproducing kernel $K_{S}(z, w)$ can be expressed directly in terms of the colligation matrix $\mathbf{U}_{S}$ :

$$
\frac{I-S(z) S(w)^{*}}{1-z \bar{w}}=C_{S}\left(I-z A_{S}\right)^{-1}\left(I-\bar{w} A_{S}^{*}\right)^{-1} C_{S}^{*} .
$$

Moreover:

(1) The following are equivalent:

(a) $\mathbf{U}_{S}$ is unitary.

(b) $S$ satisfies the condition

$$
S(z) u \in \mathcal{H}(S) \Rightarrow u=0 .
$$

(c) The maximal factorable minorant of $I-S^{*} S$ is zero, i.e., if $\Phi \in \mathcal{S}\left(\mathcal{U}, \mathcal{Y}_{0}\right)$ satisfies $\Phi(\zeta)^{*} \Phi(\zeta) \leq I-S(\zeta)^{*} S(\zeta)$ for almost all $\zeta \in \mathbb{T}$, then $\Phi=0$.

(2) The following are equivalent:

(a) The difference quotient identity holds, i.e., equality holds in (2.3).

(b) $S$ satisfies the condition

$$
S(z) u \in \mathcal{H}(S) \Rightarrow S(z) u \equiv 0 .
$$

(c) If $S^{\prime}$ is the normalization of $S$ as in (3.17) (so $S^{\prime}$ satisfies (3.17) and $\mathcal{H}(S)=\mathcal{H}\left(S^{\prime}\right)$ ), then the maximal factorable minorant of $S^{\prime *} S^{\prime}$ is zero. 
Proof. The coisometry property of $\mathbf{U}_{S}$ amounts to the three identities

$$
\begin{aligned}
R_{0} R_{0}^{*}+\tau \tau^{*} & =I_{\mathcal{H}(S)}, \\
\mathbf{e}(0) \mathbf{e}(0)^{*}+S(0) S(0)^{*} & =I_{\mathcal{Y}}, \\
\mathbf{e}(0) R_{0}^{*}+S(0) \tau^{*} & =0
\end{aligned}
$$

The identity (3.35) is the same as (2.6) which has been already verified above. From the formula (3.30) it is immediate that

$$
\mathbf{e}(0) \mathbf{e}(0)^{*}: y \mapsto \mathbf{e}(0)\left(I-S(z) S(0)^{*}\right) y=\left(I-S(0) S(0)^{*}\right) y
$$

and (3.36) now follows. Next, use the formula (2.5) for $R_{0}^{*}$ to compute

$$
\mathbf{e}(0) R_{0}^{*}: f(z) \mapsto-S(0) \tau^{*}(f)
$$

from which (3.37) is now immediate. This completes the verification of the coisometry property of $\mathbf{U}_{S}$.

To verify (2.8), let $S(z)=\sum_{n=0}^{\infty} S_{n} z^{n}$ be the Taylor series for $S(z)$. Then the computation

$$
\begin{aligned}
\left(D_{S}+w C_{S}\left(I-w A_{S}\right)^{-1} B_{S}\right) u & =S(0) u+w \sum_{n=1}^{\infty} w^{n} \mathbf{e}(0) \mathbf{S}_{\mathcal{Y}}^{* n}\left(\sum_{k=1}^{\infty} S_{k+1} z^{k}\right) u \\
& =S(0) u+w \sum_{n=1}^{\infty} w^{n} S_{n+1} u=S(w) u
\end{aligned}
$$

verifies the realization formula (3.31). The formula (3.32) can be verified by direct computation: plug in the formula (3.31) for $S(z)$ and use that $\mathbf{U}_{S}$ is coisometric.

Once it is known that $\mathbf{U}_{S}$ is coisometric, it follows that $\mathbf{U}_{S}$ is unitary if and only if $\operatorname{Ker} \mathbf{U}_{S}=\{0\}$. Note that $\left[\begin{array}{c}f(z) \\ u\end{array}\right]$ being in Ker $\mathbf{U}_{S}$ means that

$$
\left[\begin{array}{l}
0 \\
0
\end{array}\right]=\mathbf{U}_{S}\left[\begin{array}{c}
f(z) \\
u
\end{array}\right]=\left[\begin{array}{c}
{[f(z)-f(0)] / z+[S(z) u-S(0) u] / z} \\
f(0)+S(0) u
\end{array}\right] .
$$

Thus

$f(z)=f(0)+z \cdot \frac{f(z)-f(0)}{z}=-S(0) u-z \cdot \frac{S(z)-S(0)}{z} u=-S(z) u$.

This completes the proof of the equivalence of (a) and (b) in part (1) in the theorem.

A computation of Nikolskii-Vasyunin (see [NV1986, Theorem 8.7]) gives the following: for $f \in \mathcal{H}(S)$ of the special form $f=\left(I-T_{S} T_{S}^{*}\right) f_{1}$,

$$
\begin{aligned}
& \|f\|_{\mathcal{H}(S)}^{2}-\left\|R_{0} f\right\|_{\mathcal{H}(S)}^{2} \\
& \quad=\|f(0)\|^{2}+\inf \left\{\left\|\left(I-T_{S}^{*} T_{S}\right)^{\frac{1}{2}}\left(T_{S}^{*} f_{1}\right)(0)+\mathbf{S}_{\mathcal{Y}} g\right\|_{H^{2}(\mathcal{U}}^{2}: g \in H^{2}(\mathcal{U})\right\} .
\end{aligned}
$$


Therefore the operator $\left[\begin{array}{l}A_{S} \\ C_{S}\end{array}\right]=\left[\begin{array}{c}R_{0} \\ \mathbf{e}(0)\end{array}\right]$ is isometric if and only if $\inf \left\{\left\|\left(I-T_{S}^{*} T_{S}\right)^{\frac{1}{2}}\left(T_{S}^{*} f_{1}\right)(0)+\mathbf{S}_{\mathcal{Y}} g\right\|_{H^{2}(\mathcal{U}}^{2}: g \in H^{2}(\mathcal{U})\right\}=0$ for all $g \in H^{2}(\mathcal{U})$.

Another computation of Nikolskii-Vasyunin (see [NV1986, Lemma i.2]) gives:

$\|\tau(u)\|_{\mathcal{H}(S)}^{2}=\|u\|_{\mathcal{U}}^{2}-\|S(0) u\|_{\mathcal{Y}}^{2}-\inf \left\{\left\|\left(I-T_{S}^{*} T_{S}\right)^{\frac{1}{2}}\left(u+\mathbf{S}_{\mathcal{U}} g\right)\right\|^{2}: g \in H^{2}(\mathcal{U})\right\}$.

Therefore the operator $\left[\begin{array}{l}B_{S} \\ D_{S}\end{array}\right]=\left[\begin{array}{c}\tau \\ S(0)\end{array}\right]$ is isometric if and only if

$$
\inf \left\{\left\|\left(I-T_{S}^{*} T_{S}\right)^{\frac{1}{2}}\left(u+\mathbf{S}_{\mathcal{U}} g\right)\right\|^{2}: g \in H^{2}(\mathcal{U})\right\}=0 \text { for all } u \in \mathcal{U} .
$$

Note that condition (3.40) implies (3.38). The condition (3.40) amounts to the statement that vector analytic polynomials in $z$ are dense in the weighted space $L^{2}(\mathcal{U})$ with $\left(I-S(\zeta)^{*} S(\zeta)\right)|d \zeta|$-metric. It is well known how this condition in turn translates to 0 is the maximal factorable minorant for $I-S(\zeta)^{*} S(\zeta)$ (see e.g. [SNF1970, Proposition V.4.2]). In this way one can see that the zero maximal-factorable-minorant condition is equivalent to each column $\left[\begin{array}{l}A_{S} \\ C_{S}\end{array}\right]$ and $\left[\begin{array}{l}B_{S} \\ D_{S}\end{array}\right]$ of the colligation matrix $\mathbf{U}_{S}$ being isometric. As the isometry property of $U_{S}^{*}$ has already been verified above, it follows that $\mathbf{U}_{S}$ is contractive. The next elementary exercise is to verify in general that a contractive $2 \times 2$ block operator matrix $\mathbf{U}_{S}=\left[\begin{array}{cc}A_{S} & B_{S} \\ C_{S} & D_{S}\end{array}\right]$ with each column isometric must itself be isometric. In this way the equivalence of (a) and (c) in statement (1) of the theorem follows, and (1) follows as well.

It remains to verify the equivalence of (a) and (c) with the normalization assumption (3.17) imposed. For simplicity $S$ rather than $S^{\prime}$ is written with the assumption that $S$ satisfies (3.17). Then one can show that the set of elements in $\mathcal{U}$ of the form $\left(T_{S}^{*} f_{1}\right)(0)$ with $f_{1} \in H^{2}(\mathcal{Y})$ is dense in $\mathcal{U}$. Then a limiting argument implies that equality holding in (2.3) for all $f \in \mathcal{H}(S)$ is equivalent to the condition (3.40). As explained in the previous paragraph, (3.40) is equivalent to the zero maximal-factorable-minorant condition. The reverse implication follows by reversing the argument.

Remark 3.13. The equivalence of (a) and (c) in part (2) of Theorem 3.12 was already observed in [BK1987, Theorem 6]. The idea of the proof is as follows. If $\Phi^{*} \Phi$ is the maximal factorable minorant for $I-S^{*} S$ (with $\Phi$ outer), then there is a unitary transformation $J: \mathcal{H}(S) \rightarrow \mathbb{K}\left(\left[\begin{array}{l}S \\ \Phi\end{array}\right]\right)$, where $\mathbb{K}\left(\left[\begin{array}{c}S \\ \Phi\end{array}\right]\right)$ is the Sz.-Nagy-Foias model space based on the Schur-class function $\left[\begin{array}{c}S \\ \Phi\end{array}\right]$ which intertwines the de BrangesRovnyak model operator $R_{0}$ with the adjoint of the Sz.-Nagy-Foias 
model operator $\left(T_{\left[\begin{array}{l}S \\ \Phi\end{array}\right]}\right)^{*}$; on kernel functions the map $J$ has the form

$$
J: K_{S}(\cdot, w) y \mapsto\left[\begin{array}{c}
K_{S}(\cdot, w) y \\
-\Phi S(w)^{*} k_{\mathrm{Sz}}(\cdot, w) y \\
-\nabla S(w)^{*} k_{\mathrm{Sz}}(\cdot w) y
\end{array}\right]
$$

where

$$
\nabla(\zeta)=\left(I-S(\zeta)^{*} S(\zeta)-\Phi(\zeta)^{*} \Phi(\zeta)\right)^{\frac{1}{2}} \text { for } \zeta \in \mathbb{T}
$$

Note that the first two components of elements of $\mathbb{K}\left(\left[\begin{array}{c}S \\ \Phi\end{array}\right]\right)$ are analytic functions functions on $\mathbb{D}$ while the third component is a measurable function on $\mathbb{T}$, and that $J$ has the form

$$
J: f \mapsto\left[\begin{array}{c}
f \\
g_{f} \\
w_{f}
\end{array}\right],
$$

i.e., the projection of $J$ to the first component is the identity map. One next observes that

$$
\left\|R_{0} f\right\|_{\mathcal{H}(S)}^{2}=\left\|\left(T_{\left[\begin{array}{c}
S \\
\Phi
\end{array}\right]}\right)^{*}\left[\begin{array}{c}
f \\
g_{f} \\
w_{f}
\end{array}\right]\right\|^{2}=\|f\|_{\mathcal{H}(S)}^{2}-\|f(0)\|^{2}-\left\|g_{f}(0)\right\|^{2} .
$$

Hence the difference quotient identity (equality in (2.3)) holding for all $f \in \mathcal{H}(S)$ is equivalent to $g_{f}(0)=0$ for all $f \in \mathcal{H}(S)$. Under the assumption (3.17), it can be shown that this is equivalent to the normalized version $S^{\prime}$ of $S$ (i.e., $S^{\prime}$ satisfying (3.17) while $\mathcal{H}(S)=$ $\mathcal{H}\left(S^{\prime}\right)$ ) having maximal factorable minorant equal to 01

The equivalence of (a) and (c) in part (1) of Theorem 3.12 also follows from results of [BK1987]. For this alternative proof, the following fact will be used: the colligation matrix $\mathbf{U}_{S}$ being unitary is equivalent to the isometric embedding of $\mathcal{H}(S)$ into the two-component space $\mathcal{D}(S)$ being onto. Theorem 8 of [BK1987] shows that this happens if and only if the maximal factorable minorant of $I-S^{*} S$ is zero.

To this point the operator-range characterization of $\mathcal{H}(S)$ has been used to develop the basic properties of the operators $R_{0}, \tau, \mathbf{e}(0), S(0)$ in the colligation matrix $\mathbf{U}_{S}$. Alternatively, the space $\mathcal{H}(S)$ could have been defined as the RKHS with reproducing kernel $K_{S}$ and this characterization then used to obtain the results concerning $\mathbf{U}_{S}$. To see directly that $K_{S}$ is a positive kernel (without recourse to the operatorrange characterization of $\mathcal{H}(S)$ ), it suffices to note that the Toeplitz

\footnotetext{
${ }^{1}$ This last point was missed in BK1987; the normalization condition (3.17) was not mentioned explicitly.
} 
operator $T_{S}$ has $\left\|T_{S}\right\| \leq 1$ for $S \in \mathcal{S}(\mathcal{U}, \mathcal{Y}$ ) (since the boundary-value function $\zeta \mapsto S(\zeta)$ on $\mathbb{T}$ has contractive values) and hence

$$
\|f\|_{H^{2}(\mathcal{Y})}^{2}-\left\|T_{S}^{*} f\right\|_{H^{2}(\mathcal{U})}^{2} \geq 0 \quad \text { for all } \quad f \in H^{2}(\mathcal{Y}) .
$$

Set $f=\sum_{j=1}^{N} k_{\mathrm{Sz}}\left(\cdot, w_{j}\right) y_{j} \in H^{2}(\mathcal{Y})$. Then condition (3.41) translates to (3.25), and it follows that $K_{S}$ is a positive kernel and hence one can define $\mathcal{H}(S)$ as the reproducing kernel Hilbert space $\mathcal{H}\left(K_{S}\right)$. The following discussion presents an alternate proof of parts (2), (3), and (4) of Theorem 2.1 using the reproducing-kernel-space characterization (i.e., part (1) of Theorem 2.1) rather than the operator-range characterization (3.18) of the space $\mathcal{H}(S)$.

Proof of parts (2), (3), (4) of Theorem 2.1 based on part (1). Given that $S$ is in the Schur class $\mathcal{S}(\mathcal{U}, \mathcal{Y})$, it has been explained in the previous paragraph why $K_{S}$ is a positive kernel (i.e., satisfies (3.25)) and hence generates a well-defined RKHS $\mathcal{H}\left(K_{S}\right)$. By the general theory of RKHSs (see (3.26) above and the explanation there), it is known that $K_{S}$ has its canonical Kolmogorov decomposition

$$
K_{S}(z, w)=\mathbf{e}(z) \mathbf{e}(w)^{*}
$$

where $\mathbf{e}(z): \mathcal{H}\left(K_{S}\right) \rightarrow \mathcal{Y}$ is the point-evaluation map $\mathbf{e}(z): f \mapsto f(z)$ for each $z \in \mathbb{D}$. Substituting $K_{S}(z, w)=\left[I-S(z) S(w)^{*}\right] /(1-z \bar{w})$ and rearranging (3.42) leads to

$$
z \bar{w} \mathbf{e}(z) \mathbf{e}(w)^{*}+I_{\mathcal{Y}}=\mathbf{e}(z) \mathbf{e}(w)^{*}+S(z) S(w)^{*} .
$$

The inner-product identity

$$
\left\langle\bar{w} \mathbf{e}(w)^{*} y, \bar{z} \mathbf{e}(z)^{*} y^{\prime}\right\rangle_{\mathcal{H}\left(K_{S}\right)}+\left\langle y, y^{\prime}\right\rangle_{\mathcal{Y}}=\left\langle\mathbf{e}(w)^{*} y, \mathbf{e}(z)^{*} y^{\prime}\right\rangle_{\mathcal{H}(K)}+\left\langle S(w)^{*} y, S(z)^{*} y^{\prime}\right\rangle_{\mathcal{U}}
$$

then follows, where $y, y^{\prime}$ are arbitrary vectors in $\mathcal{Y}$. This inner-product identity can be written in aggregate form

$$
\left\langle\left[\begin{array}{c}
\bar{w} \mathbf{e}(w)^{*} \\
I
\end{array}\right] y,\left[\begin{array}{c}
\bar{z} \mathbf{e}(z)^{*} \\
I
\end{array}\right] y^{\prime}\right\rangle_{\mathcal{H}\left(K_{S}\right) \oplus \mathcal{Y}}=\left\langle\left[\begin{array}{l}
\mathbf{e}(w)^{*} \\
S(w)^{*}
\end{array}\right] y,\left[\begin{array}{l}
\mathbf{e}(z)^{*} \\
S(z)^{*}
\end{array}\right] y^{\prime}\right\rangle_{\mathcal{H}\left(K_{S}\right) \oplus \mathcal{U}} .
$$

It follows that the mapping

$$
V:\left[\begin{array}{c}
\bar{w} \mathbf{e}(w)^{*} \\
I
\end{array}\right] y \mapsto\left[\begin{array}{l}
\mathbf{e}(w)^{*} \\
S(w)^{*}
\end{array}\right] y
$$

extends by linearity and continuity to an isometry from

$$
\mathcal{D}=\overline{\operatorname{span}}\left\{\left[\begin{array}{c}
\bar{w} \mathbf{e}(w)^{*} \\
I
\end{array}\right] y: w \in \mathbb{D}, y \in \mathcal{Y}\right\}
$$

onto

$$
\mathcal{R}=\overline{\operatorname{span}}\left\{\left[\begin{array}{l}
\mathbf{e}(w)^{*} \\
S(w)^{*}
\end{array}\right] y: w \in \mathbb{D}, y \in \mathcal{Y}\right\}
$$


Taking $w=0$ in the expression for a given element of $\mathcal{D}$ reveals that $\mathcal{D} \supset\left[\begin{array}{c}\{0\} \\ \mathcal{Y}\end{array}\right]$. Since the kernel elements $\left\{\mathbf{e}(w)^{*} y=K_{S}(\cdot, w) y: w \in \mathbb{D} \backslash\right.$ $\{0\}, y \in \mathcal{Y}\}$ are dense in $\mathcal{H}\left(K_{S}\right)$, it next follows that in fact $\mathcal{D}$ is the whole space $\mathcal{D}=\left[\begin{array}{c}\mathcal{H}\left(K_{S}\right) \\ \mathcal{Y}\end{array}\right]$ and $V$ is defined on the whole space $\mathcal{D}=\left[\begin{array}{c}\mathcal{H}\left(K_{S}\right) \\ \mathcal{Y}\end{array}\right]$. Write out $V$ in block-matrix form

$$
V=\left[\begin{array}{ll}
A^{*} & C^{*} \\
B^{*} & D^{*}
\end{array}\right]:\left[\begin{array}{c}
\mathcal{H}\left(K_{S}\right) \\
\mathcal{Y}
\end{array}\right] \rightarrow\left[\begin{array}{c}
\mathcal{H}\left(K_{S}\right) \\
\mathcal{U}
\end{array}\right]
$$

It then follows from (3.43) that

$$
\left[\begin{array}{ll}
A^{*} & C^{*} \\
B^{*} & D^{*}
\end{array}\right]:\left[\begin{array}{c}
\bar{w} \mathbf{e}(w)^{*} \\
I
\end{array}\right] y=\left[\begin{array}{l}
\mathbf{e}(w)^{*} \\
S(w)^{*}
\end{array}\right] y
$$

or

$$
\left\{\begin{array}{l}
\bar{w} A^{*} \mathbf{e}(w)^{*} y+C^{*} y=\mathbf{e}(w)^{*} y \\
\bar{w} B^{*} \mathbf{e}(w)^{*} y+D^{*} y=S(w)^{*} y
\end{array}\right.
$$

The first equation can be solved for $\mathbf{e}(w)^{*} y$ (note that $\|A\| \leq 1$ since $V$ is isometric and hence the inverse $\left(I-\bar{w} A^{*}\right)^{-1}$ is well-defined for all $w \in \mathbb{D})$ :

$$
\mathbf{e}(w)^{*} y=\left(I-\bar{w} A^{*}\right)^{-1} C^{*} y .
$$

The second equation then implies

$$
\left(\bar{w} B^{*}\left(I-\bar{w} A^{*}\right)^{-1} C^{*}+D^{*}\right) y=S(w)^{*} y .
$$

Cancelling off the vector $y$, taking adjoints, and replacing the variable $w$ with the variable $z$ then gives

$S(z)=D+z C(I-z A)^{-1} B \quad$ where $\quad\left[\begin{array}{ll}A & B \\ C & D\end{array}\right]=V^{*} \quad$ is a coisometry.

Putting the pieces together leads to part (4) of Theorem 2.1 apart from making the identification $V^{*}=\mathbf{U}_{S}$.

Letting $w=0$ in (3.44) enables one to solve for $C^{*}$ :

$$
C^{*} y=\mathbf{e}(0)^{*} y=K_{S}(\cdot, 0) y .
$$

The simple duality computation

$$
\langle C f, y\rangle_{\mathcal{Y}}=\left\langle f, C^{*} y\right\rangle_{\mathcal{H}\left(K_{S}\right)}=\left\langle f, K_{S}(\cdot, 0) y\right\rangle_{\mathcal{H}\left(K_{S}\right)}=\langle f(0), y\rangle_{\mathcal{Y}}
$$

shows that $C=\mathbf{e}(0)$. One can use (3.44) to compute the action of $A^{*}$ on kernel elements $\mathbf{e}(w)^{*} y$ as follows:

$$
A^{*} \mathbf{e}(w)^{*} y=\frac{1}{\bar{w}}\left(\mathbf{e}(w)^{*}-\mathbf{e}(0)^{*}\right) y
$$


Another duality computation

$$
\begin{aligned}
\left\langle A f, \mathbf{e}(w)^{*} y\right\rangle_{\mathcal{H}\left(K_{S}\right)} & =\left\langle f, A^{*} \mathbf{e}(w)^{*} y\right\rangle_{\mathcal{H}\left(K_{S}\right)} \\
& =\left\langle f, \frac{1}{\bar{w}}\left(\mathbf{e}(w)^{*}-\mathbf{e}(0)^{*}\right) y\right\rangle_{\mathcal{H}\left(K_{S}\right)} \\
& =\langle[f(w)-f(0)] / w, y\rangle_{\mathcal{Y}}
\end{aligned}
$$

leads to the conclusion that

$$
A: f(z) \mapsto[f(z)-f(0)] / z,
$$

i.e., that $A=R_{0}=\left.\mathbf{S}_{\mathcal{Y}}^{*}\right|_{\mathcal{H}\left(K_{S}\right)}$. Application of the second equation in (3.44) with $w=0$ yields that $D^{*}=S(0)^{*}$, i.e., $D=S(0)$. A return to the second equation in (3.44) then implies that

$$
B^{*} \mathbf{e}(w)^{*} y \mapsto\left(\left[S(w)^{*}-S(0)^{*}\right] / \bar{w}\right) y .
$$

Then the computation

$$
\begin{aligned}
\left\langle B u, \mathbf{e}(w)^{*} y\right\rangle_{\mathcal{H}\left(K_{S}\right)} & =\left\langle u,\left[\left(S(w)^{*}-S(0)^{*}\right] / \bar{w}\right) y\right\rangle_{\mathcal{U}} \\
& =\langle([S(w)-S(0)] / w) u, y\rangle_{\mathcal{Y}}
\end{aligned}
$$

verifies that $B: u \mapsto([S(z)-S(0)] / z) u$, i.e., $B=\tau$ where $\tau$ is as in (2.4). It has been now verified that $\left[\begin{array}{ll}A & B \\ C & D\end{array}\right]=\mathbf{U}_{S}$. Moreover (2.3) and (2.6) are immediate consequences of the already derived property that $\mathbf{U}_{S}=\left[\begin{array}{cc}A & B \\ C & D\end{array}\right]$ is a coisometry.

Remark 3.14. The above construction has become known as the lurking isometry argument (see [B2000] where this term was first coined). In this single-variable setting, it turns out that the isometry is defined on the whole space; in other applications (see [B2000]), the isometry is defined only on a subspace and one must extend it to an isometry (or unitary or contraction depending on what is wanted) on the whole space.

The preparation is now laid for the use RKHS techniques to prove part (5) of Theorem 2.1. The following is an enhanced version of the necessity direction of part (5) of Theorem 2.1; note that the sufficiency direction is handled in Theorem 3.9 above. The following proof is based on the RKHS characterization of $\mathcal{H}(S)$ (part (1) of Theorem 2.1).

Theorem 3.15. Let $A$ be a completely non-isometric contraction operator on a Hilbert space $\mathcal{X}$. Then there is a Schur-class function $S \in \mathcal{S}(\mathcal{U}, \mathcal{Y})$ so that $T$ is unitarily equivalent to the model operator $R_{0}=\left.\mathbf{S}_{\mathcal{Y}}^{*}\right|_{\mathcal{H}(S)}$ on $\mathcal{H}(S)$. Furthermore one can arrange that $I-S^{*} S$ have maximal factorable minorant equal to 0. 
Proof. Let $A$ be any completely non-isometric contraction operator on a Hilbert space $\mathcal{X}$. Choose an operator $C$ from $\mathcal{X}$ to a coefficient Hilbert space $\mathcal{Y}$ so that the block column operator $\left[\begin{array}{l}A \\ C\end{array}\right]: \mathcal{X} \rightarrow\left[\begin{array}{l}\mathcal{X} \\ \mathcal{Y}\end{array}\right]$ is an isometry, i.e., so that

$$
C^{*} C=I_{\mathcal{X}}-A^{*} A .
$$

Note that one way to do this is to take $\mathcal{Y}=\mathcal{D}_{A}$ equal to the defect space of $A$

$$
\mathcal{D}_{A}=\overline{\operatorname{Ran}} D_{A}
$$

where $D_{A}$ is the defect operator of $A$ :

$$
D_{A}=\left(I-A^{*} A\right)^{\frac{1}{2}} \text {. }
$$

Consider the operator $\mathcal{O}_{C, A}: \mathcal{X} \rightarrow H^{2}(\mathcal{Y})$ defined by

$$
\mathcal{O}_{C, A}: x \mapsto C(I-z A)^{-1} x .
$$

The notation $\mathcal{O}_{C, A}(z)=C(I-z A)^{-1}$ is useful; then $\left(\mathcal{O}_{C, A} x\right)(z)=$ $\mathcal{O}_{C, A}(z) x$. To see that $\mathcal{O}_{C, A}$ maps into $H^{2}(\mathcal{Y})$, note that $\mathcal{O}_{C, A} x$ has Taylor series

$$
\mathcal{O}_{C, A}(z) x=\sum_{n=0}^{\infty}\left(C A^{n} x\right) z^{n} .
$$

The computation of the $H^{2}(\mathcal{Y})$-norm of $\mathcal{O}_{C, A} x$ can be organized as follows:

$$
\begin{aligned}
\left\|\mathcal{O}_{C, A} x\right\|_{H^{2}(\mathcal{Y})}^{2} & =\sum_{n=0}^{\infty}\left\|C A^{n} x\right\|_{\mathcal{Y}}^{2}=\sum_{n=0}^{\infty}\left\langle A^{* n} C^{*} C A^{n} x, x\right\rangle_{\mathcal{X}} \\
& =\sum_{n=0}^{\infty}\left\langle A^{* n}\left(I-A^{*} A\right) A^{n} x, x\right\rangle_{\mathcal{X}}=\sum_{n=0}^{\infty}\left[\left\|A^{n} x\right\|_{\mathcal{X}}^{2}-\left\|A^{n+1} x\right\|_{\mathcal{X}}^{2}\right] \\
& =\|x\|_{\mathcal{X}}^{2}-\lim _{N \rightarrow \infty}\left\|A^{N} x\right\|_{\mathcal{X}}^{2}<\infty
\end{aligned}
$$

(where the limit exists since the sequence $\left\{\left\|A^{N} x\right\|\right\}_{N \geq 0}$ is nonincreasing). For reasons connected with system theory which are not discussed here, the notation $\mathcal{O}_{C, A}$ is sued to suggest the observability operator for the output pair $(C, A)$. Note that $x \in \operatorname{Ker} \mathcal{O}_{C, A}$ if and only if $C A^{n} x=0$ for $n=0,1,2, \ldots$, or equivalently, if and only if

$0=\left\langle A^{* n} C^{*} C A^{n} x, x\right\rangle_{\mathcal{X}}=\left\langle A^{*}\left(I-A^{*} A\right) A^{n} x, x\right\rangle=\left\|A^{n} x\right\|_{\mathcal{X}}^{2}-\left\|A^{n+1} x\right\|_{\mathcal{X}}^{2}$.

Thus $\left\|A^{n} x\right\|=\|x\|$ for all $n=1,2, \ldots$, or $\operatorname{Ker} \mathcal{O}_{C, A}$ is the maximal invariant subspace for $A$ on which $A$ is isometric. The assumption that $A$ is completely non-isometric implies that $x=0$, and hence the 
observability operator $\mathcal{O}_{C, A}$ is one-to-one. Define the Hilbert space $\mathcal{H}_{0}$ as $\mathcal{H}_{0}=\operatorname{Ran} \mathcal{O}$ with the lifted norm:

$$
\left\|\mathcal{O}_{C, A} x\right\|_{\mathcal{H}_{0}}=\|x\|_{\mathcal{X}}
$$

i.e., $\mathcal{H}_{0}$ is the pullback space $\mathcal{H}_{0}=\mathcal{H}_{\mathcal{O}_{C, A}}^{p}$. The computation

$$
\mathbf{S}_{\mathcal{Y}}^{*} \mathcal{O}_{C, A} x=\sum_{n=0}^{\infty}\left(C A^{n+1} x\right) z^{n}=\left(\sum_{n=0}^{\infty} C A^{n} x z^{n}\right) A=\mathcal{O}_{C, A}(A x)
$$

verifies the intertwining relation

$$
\mathbf{S}_{\mathcal{Y}}^{*} \mathcal{O}_{C, A}=\mathcal{O}_{C, A} A
$$

As by definition $\mathcal{O}_{C, A}$ is a unitary transformation from $\mathcal{X}$ onto $\mathcal{H}_{0}$, it follows that $A$ is unitarily equivalent to the operator $R_{0} \in \mathcal{L}\left(\mathcal{H}_{0}\right)$ given by $R_{0}=\left.\mathbf{S}_{\mathcal{Y}}^{*}\right|_{\mathcal{H}_{0}}$.

It remains to identify the space $\mathcal{H}_{0}$ more explicitly as a RKHS. Since $\mathcal{H}_{0}$ sits inside $H^{2}(\mathcal{Y})$, the point-evaluation maps $f \mapsto f(w)$ are welldefined for all $w \in \mathbb{D}$. Therefore

$$
\begin{aligned}
\langle f(w), y\rangle_{\mathcal{Y}} & =\left\langle C(I-w A)^{-1} x, y\right\rangle_{\mathcal{Y}}=\left\langle x,\left(I-\bar{w} A^{*}\right)^{-1} y\right\rangle_{\mathcal{X}} \\
& =\left\langle\mathcal{O}_{C, A} x, \mathcal{O}_{C, A}\left(I-\bar{w} A^{*}\right)^{-1} y\right\rangle_{\mathcal{H}_{0}} \\
& =\left\langle f, \mathcal{O}_{C, A}\left(I-\bar{w} A^{*}\right)^{-1} C^{*}\right\rangle_{\mathcal{H}_{0}}
\end{aligned}
$$

where

$$
\left(\mathcal{O}_{C, A}\left(I-\bar{w} A^{*}\right)^{-1} C^{*} y\right)(z)=C(I-z A)^{-1}\left(I-\bar{w} A^{*}\right)^{-1} C^{*} y=: K_{C, A}(z, w) y .
$$

It now follows that $\mathcal{H}_{0}$ is a RKHS with reproducing kernel equal to $K_{C, A}$. The claim to be checked now is that $K_{C, A}$ in fact has the form $K_{S}(z, w)=\left[I-S(z) S(w)^{*}\right] /(1-z \bar{w})$ for a Schur-class function $S \in$ $\mathcal{S}(\mathcal{U}, \mathcal{Y})$ for an appropriate coefficient Hilbert space $\mathcal{U}$.

Toward this end, a useful observation is that the formula (3.32) in Theorem 3.12 is quite general: if $\mathbf{U}=\left[\begin{array}{ll}A & B \\ C & D\end{array}\right]:\left[\begin{array}{l}\mathcal{X} \\ \mathcal{U}\end{array}\right] \rightarrow\left[\begin{array}{l}\mathcal{X} \\ \mathcal{Y}\end{array}\right]$ is coisometric. The function $S$ given by $S(z)=D+z C(I-z A)^{-1} B$ is in the Schur class $\mathcal{S}(\mathcal{U}, \mathcal{Y})$ and

$$
\frac{I-S(z) S(w)^{*}}{1-z \bar{w}}=C(I-z A)^{-1}\left(I-\bar{w} A^{*}\right)^{-1} C^{*}=K_{C, A}(z, w) .
$$

Thus, identification of $K_{C, A}$ as having the form $K_{S}$ requires only a solution of the matrix completion problem: given the isometric output pair $(C, A)$ (so $A^{*} A+C^{*} C=I$ ), find a block-column operator matrix $\left[\begin{array}{l}B \\ D\end{array}\right]$ so that the operator matrix $\mathbf{U}=\left[\begin{array}{ll}A & B \\ C & D\end{array}\right]$ is coisometric. But this is 
easily done by solving a Cholesky factorization problem: find $\left[\begin{array}{l}B \\ D\end{array}\right]$ so that

$$
\left[\begin{array}{l}
B \\
D
\end{array}\right]\left[\begin{array}{ll}
B^{*} & D^{*}
\end{array}\right]=\left[\begin{array}{ll}
I & 0 \\
0 & I
\end{array}\right]-\left[\begin{array}{l}
A \\
C
\end{array}\right]\left[\begin{array}{ll}
A^{*} & C^{*}
\end{array}\right] .
$$

Given that $\left[{ }_{C}^{A}\right]$ is isometric, it is not difficult to see that this problem is solvable; in fact, one can arrange that $\left[\begin{array}{l}B \\ D\end{array}\right]$ is injective and then $\mathbf{U}$ will be unitary (not just coisometric). Furthermore, an adaptation of the lurking isometry argument (see Remark 3.14) in the proof of part (4) of Theorem 2.1 above shows that the colligation matrix $\mathbf{U}$ is unitarily equivalent to the model colligation matrix $\mathbf{U}_{S}$ (see BB2010 for precise details). As a consequence of part (1) of Theorem 2.1, the conclusion that $S$ has maximal factorable minorant equal to 0 follows. This completes the proof of Theorem 3.15.

Remark 3.16. Let $S \in \mathcal{S}(\mathcal{U}, \mathcal{Y})$ be any Schur-class function (possibly with nonzero maximal factorable minorant) and let $A$ be the model operator $A=R_{0}=\left.\mathbf{S}_{\mathcal{Y}}^{*}\right|_{\mathcal{H}(S)}$ on $\mathcal{H}(S)$. Theorem 3.9 tells us that $A$ is completely non-isometric. Therefore one can apply the construction of Theorem 3.15 to arrive at a Schur-class function $S_{0} \in \mathcal{S}\left(\mathcal{U}_{0}, \widetilde{\mathcal{Y}}_{0}\right)$ so that $A$ is unitarily equivalent to $R_{0}=\left.\mathbf{S}_{\mathcal{Y}_{0}}^{*}\right|_{\mathcal{H}\left(S_{0}\right)}$ on $\mathcal{H}\left(S_{0}\right)$ where $S_{0}$ has the additional property that $I-S_{0}^{*} S_{0}$ has zero maximal factorable minorant. Since the original $S$ for which $I-S^{*} S$ does not have zero maximal factorable minorant, it cannot be the case that $S$ and $S_{0}$ are the same. A natural question is: how does $S$ determine $S_{0}$ ? As can be seen from the results of [BK1987] (details left to the reader), the answer is: one choice of $S_{0}$ is $S_{0}=\left[\begin{array}{c}S \\ \Psi\end{array}\right]$ where $\Psi$ is the outer factor for the maximal factorable minorant of $I-S^{*} S$.

Remark 3.17. The zero maximal-factorable-minorant property is also closely intertwined with the characterization of the extreme points of the closed unit ball of $H^{\infty}(\mathcal{U}, \mathcal{Y})$, i.e., of the Schur class $\mathcal{S}(\mathcal{U}, \mathcal{Y})$. In the scalar case $(\mathcal{U}=\mathcal{Y}=\mathbb{C})$, it is well known that a given function $s$ is an extreme point of $\mathcal{S}(\mathbb{C})$ exactly when $\log \left(1-|s(\zeta)|^{2}\right)$ is log-integrable on $\mathbb{T}$ which in turn is equivalent to $1-|s(\zeta)|^{2}$ having a factorization $a(\zeta)^{*} a(\zeta)$ with $a$ a nonzero element of $\mathcal{S}(\mathbb{C})$ which in turn (in the scalar case) is equivalent to 0 not being the maximal factorable minorant for $1-|s(\zeta)|^{2}$. In [BK1987] it was observed for the case of $S \in \mathcal{S}(\mathcal{U}, \mathcal{Y})$ that $I-S(\zeta)^{*} S(\zeta)$ having a zero maximal factorable minorant is a sufficient condition for $S$ to be an extreme point of $\mathcal{S}(\mathcal{U}, \mathcal{Y})$, and by symmetry it is also sufficient that $I-S(\zeta) S(\zeta)^{*}$ have a zero maximal factorable minorant. It was then conjectured there (with attribution to de Branges) that a necessary and sufficient condition for $S$ to be extreme in $\mathcal{S}(\mathcal{U}, \mathcal{Y})$ 
is that at least one of $I-S(\zeta)^{*} S(\zeta)$ and $I-S(\zeta) S(\zeta)^{*}$ have a zero maximal factorable minorant. This conjecture was independently confirmed around the same time by Treil (see [T1989]).

In Section [3.2, the overlapping space $\mathcal{L}_{T}$ was defined by formulas (3.13), (3.14) for the case of a general contraction operator $T \in$ $\mathcal{L}\left(\mathcal{H}_{1}, \mathcal{H}_{2}\right)$. Although not discussed in Section 3.3, of course this notion applies to the situation where $T=T_{S} \in \mathcal{L}\left(H^{2}(\mathcal{U}), H^{2}(\mathcal{Y})\right)$ is the analytic Toeplitz operator arising from a Schur-class function $S \in \mathcal{S}(\mathcal{U}, \mathcal{Y})$. The following result identifies the associated overlapping space $\mathcal{L}_{T_{S}}$ as a reproducing kernel Hilbert space.

Theorem 3.18. For $S \in \mathcal{S}(\mathcal{U}, \mathcal{Y})$, the overlapping space $\mathcal{L}_{T_{S}}$ defined by (3.13), (3.14) is a reproducing kernel Hilbert spaces $\mathcal{H}\left(K_{\mathcal{L}(S)}\right)$ with reproducing kernel $K_{\mathcal{L}(S)}$ given by

$$
K_{\mathcal{L}(S)}(z, w)=\frac{1}{2} \frac{\varphi(z)+\varphi(w)^{*}}{1-z \bar{w}}
$$

where $\varphi(z)$ is given by

$$
\varphi(z)=\int_{\mathbb{T}} \frac{\zeta+z}{\zeta-z}\left(I-S(\zeta)^{*} S(\zeta)\right) \frac{|\mathrm{d} \zeta|}{2 \pi} .
$$

Proof. Note first that the formula (3.47) for $\varphi$ together with straightforward algebra enables one to show that

$$
\frac{1}{2} \frac{\varphi(z)+\varphi(w)^{*}}{1-z \bar{w}}=\int_{\mathbb{T}} \frac{1}{(1-z \bar{\zeta})(1-\bar{w} \zeta)}\left(I-S(\zeta)^{*} S(\zeta)\right) \frac{|\mathrm{d} \zeta|}{2 \pi} .
$$

On the other hand, Proposition 3.5 identifies $\mathcal{L}_{T_{S}}$ as the lifted-norm space $\mathcal{H}_{I-T_{S}^{*} T_{S}}$. For $f=\left(I-T_{S}^{*} T_{S}\right) g \in \mathcal{L}_{T_{S}}$ and $u \in \mathcal{U}$ and $w \in \mathbb{D}$,

$$
\begin{aligned}
\langle f(w), u\rangle_{\mathcal{U}} & \left.=\left\langle\left(I-T_{S}\right)^{*} T_{S}\right) g, k_{\mathrm{Sz}}(\cdot, w) u\right\rangle_{H^{2}(\mathcal{U})} \\
& =\left\langle f,\left(I-T_{S}^{*} T_{S}\right) k_{\mathrm{Sz}}(\cdot, w) u\right\rangle_{H^{2}(\mathcal{U})} .
\end{aligned}
$$

As the set of all $f$ of the form $f=\left(I-T_{S}^{*} T_{S}\right) g$ is dense in $\mathcal{H}_{I-T_{S}^{*} T_{S}}^{\ell}$, it follows that the kernel function for $\mathcal{L}_{T_{S}}$ is equal to $K_{\mathcal{L}(S)}(\cdot, w) u:=$ $\left(I-T_{S}^{*} T_{S}\right) k_{\mathrm{Sz}}(\cdot, w) u$. This object can be computed as follows: for $u, u^{\prime} \in \mathcal{U}$,

$$
\begin{aligned}
\left\langle K_{\mathcal{L}(S)}(z, w) u, u^{\prime}\right\rangle_{\mathcal{U}} & =\left\langle\left(I-T_{S}^{*} T_{S}\right) k_{\mathrm{Sz}}(\cdot, w) u, k_{\mathrm{Sz}}(\cdot, z) u^{\prime}\right\rangle_{H^{2}(\mathcal{U})} \\
& =\int_{\mathbb{T}} \frac{1}{1-\zeta \bar{w}} \cdot \frac{1}{1-\overline{\zeta z}} \cdot\left\langle\left(I-S(\zeta)^{*} S(\zeta)\right) u, u^{\prime}\right\rangle_{\mathcal{U}} \frac{|\mathrm{d} \zeta|}{2 \pi} .
\end{aligned}
$$

Comparison of this expression with (3.48) and using that $|\zeta|=1$ for $\zeta \in \mathbb{T}$ leads to the conclusion that indeed $K_{\mathcal{L}(S)}$ is given by (3.46) as claimed. 


\section{The De Branges-Rovnyak SPaCE $\mathcal{D}(S)$}

This section gives a brief discussion of the de Branges-Rovnyak space $\mathcal{D}(S)$. The first point of discussion is the RKHS point of view; there follows an elaboration of the additional insights coming from viewing $\mathcal{D}(S)$ (or rather a certain minor modification) as a pullback space.

4.1. $\mathcal{D}(S)$ as a reproducing kernel Hilbert space. Given a Schurclass function $S \in \mathcal{D}(S)$, one can define a kernel $\widehat{K}_{S}$ as in (2.10). Unlike the case for $K_{S}$ (see the discussion around (3.41)), it is not immediately obvious why $\widehat{K}_{S}$ is a positive kernel. One way to see this is as follows. By the earlier discussion around (3.41), one can use the connection with Toeplitz operators acting on $H^{2}$ spaces to see that $K_{S}$ is a positive kernel. It is known that a Schur-class function $S$ has a unitary realization

$$
S(z)=D+z C(I-z A)^{-1} B \quad \text { with } \quad \mathbf{U}=\left[\begin{array}{cc}
A & B \\
C & D
\end{array}\right] \text { unitary; }
$$

one can cite the result of Sz.-Nagy-Foias [SNF1970, Theorem VI.3.1] or adapt the lurking isometry argument to get a unitary realization [B2000, Theorem 2.1]. Once this is done, it is a direct calculation using the relations associated with $\mathbf{U}$ being unitary to get the Kolmogorov decomposition for $\widehat{K}(z, w)$

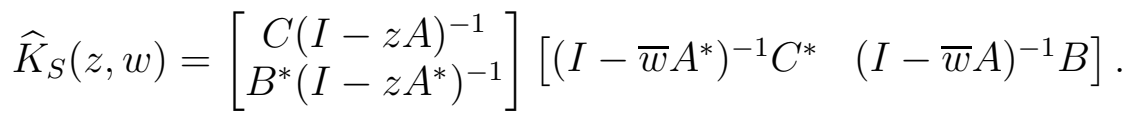

Proof of parts (2) 8 (3), (4) in Theorem 2.2 based on part (1): Once it is known that $\widehat{K}_{S}$ is a positive kernel (in this case due to the Kolmogorov decomposition (4.1)), then it is also known that $\widehat{K}_{S}$ generates a RKHS $\mathcal{D}(S)=\mathcal{H}\left(\widehat{K}_{S}\right)$ and hence has its canonical Kolmogorov decomposition

$$
\widehat{K}_{S}(z, w):=\left[\begin{array}{cc}
\frac{I-S(z) S(w)^{*}}{1-z \bar{w}} & \frac{S(z)-S(\bar{w})}{z-\bar{w}} \\
\frac{\widetilde{S}(z)-\bar{S}(\bar{w})}{z-\bar{w}} & \frac{I-\widetilde{S}(z) \widetilde{S}(\bar{w})}{1-z \bar{w}}
\end{array}\right]=\left[\begin{array}{l}
\mathbf{e}_{1}(z) \\
\mathbf{e}_{2}(z)
\end{array}\right]\left[\begin{array}{ll}
\mathbf{e}_{1}(w)^{*} & \mathbf{e}_{2}(w)^{*}
\end{array}\right]
$$

where

$$
\mathbf{e}_{1}(z):\left[\begin{array}{l}
f \\
g
\end{array}\right] \mapsto f(z), \quad \mathbf{e}_{2}(z):\left[\begin{array}{l}
f \\
g
\end{array}\right] \mapsto g(z)
$$

are the point-evaluation maps in the first and second components respectively. A rearrangement of the matricial identity (4.2) leads to the 
system of equations

$$
\begin{aligned}
z \bar{w} \mathbf{e}_{1}(z) \mathbf{e}_{1}(w)^{*}+I_{\mathcal{Y}} & =\mathbf{e}_{1}(z) \mathbf{e}_{1}(w)^{*}+S(z) S(w)^{*}, \\
\bar{w} \mathbf{e}_{2}(z) \mathbf{e}_{1}(w)^{*}+\widetilde{S}(z) & =z \mathbf{e}_{2}(z) \mathbf{e}_{1}(w)^{*}+S(w)^{*}, \\
z \mathbf{e}_{1}(z) \mathbf{e}_{2}(w)^{*}+S(\bar{w}) & =\bar{w} \mathbf{e}_{1}(z) \mathbf{e}_{2}(w)^{*}+S(z), \\
\mathbf{e}_{2}(z) \mathbf{e}_{2}(w)^{*}+S(\bar{z})^{*} S(\bar{w}) & =z \bar{w} \mathbf{e}_{2}(z) \mathbf{e}_{2}(w)^{*}+I_{\mathcal{U}}
\end{aligned}
$$

which is equivalent to the collection of inner-product identities

$$
\begin{gathered}
\left\langle\bar{w} \mathbf{e}_{1}(w)^{*} y, \bar{z} \mathbf{e}_{1}(z)^{*} y^{\prime}\right\rangle_{\mathcal{H}\left(\widehat{K}_{S}\right)}+\left\langle y, y^{\prime}\right\rangle_{\mathcal{Y}} \\
=\left\langle\mathbf{e}_{1}(w)^{*} y, \mathbf{e}_{1}(z)^{*} y^{\prime}\right\rangle_{\mathcal{H}\left(\widehat{K}_{S}\right)}+\left\langle S(w)^{*} y, S(z)^{*} y^{\prime}\right\rangle_{\mathcal{U}}, \\
\left\langle\bar{w} \mathbf{e}_{1}(w)^{*} y, \mathbf{e}_{2}(z)^{*} u^{\prime}\right\rangle_{\mathcal{H}\left(\widehat{K}_{S}\right)}+\left\langle\widetilde{S}(z) y, u^{\prime}\right\rangle_{\mathcal{U}} \\
=\left\langle\mathbf{e}_{1}(w)^{*} y, \bar{z} \mathbf{e}_{2}(z)^{*} u^{\prime}\right\rangle_{\mathcal{H}\left(\widehat{K}_{S}\right)}+\left\langle S(w)^{*} y, u^{\prime}\right\rangle_{\mathcal{U}}, \\
\left\langle\mathbf{e}_{2}(w)^{*} u, \bar{z} \mathbf{e}_{1}(z)^{*} y^{\prime}\right\rangle_{\mathcal{H}\left(\widehat{K}_{S}\right)}+\left\langle S(\bar{w}) u, y^{\prime}\right\rangle_{\mathcal{Y}} \\
=\left\langle\bar{w} \mathbf{e}_{2}(w)^{*} u, \mathbf{e}_{1}(z)^{*} y^{\prime}\right\rangle_{\mathcal{H}\left(\widehat{K}_{S}\right)}+\left\langle u, S(z)^{*} y^{\prime}\right\rangle_{\mathcal{U}}, \\
\left\langle\mathbf{e}_{2}(w)^{*} u, \mathbf{e}_{2}(z)^{*} u^{\prime}\right\rangle_{\mathcal{H}\left(\widehat{K}_{S}\right)}+\left\langle S(\bar{w}) u, S(\bar{z}) u^{\prime}\right\rangle_{\mathcal{Y}} \\
=\left\langle\bar{w} \mathbf{e}_{2}(w)^{*} u, \bar{z}_{2}(z)^{*} u^{\prime}\right\rangle_{\mathcal{H}\left(\widehat{K}_{S}\right)}+\left\langle u, u^{\prime}\right\rangle_{\mathcal{U}} .
\end{gathered}
$$

These in turn can be rearranged in aggregate form

$$
\begin{gathered}
\left\langle\left[\begin{array}{c}
\bar{w} \mathbf{e}_{1}(w)^{*} y+\mathbf{e}_{2}(w)^{*} u \\
y+S(\bar{w}) u
\end{array}\right],\left[\begin{array}{c}
\bar{z} \mathbf{e}_{1}(z)^{*} y^{\prime}+\mathbf{e}_{2}(z)^{*} u^{\prime} \\
y^{\prime}+S(\bar{z}) u^{\prime}
\end{array}\right]\right\rangle_{\mathcal{H}\left(\widehat{K}_{S}\right) \oplus \mathcal{Y}} \\
=\left\langle\left[\begin{array}{c}
\mathbf{e}_{1}(w)^{*} y+\bar{w} \mathbf{e}_{2}(w)^{*} u \\
S(w)^{*} y+u
\end{array}\right],\left[\begin{array}{c}
\mathbf{e}_{1}(w)^{*} y^{\prime}+\bar{z} \mathbf{e}_{2}(z)^{*} u^{\prime} \\
S(z)^{*} y^{\prime}+u^{\prime}
\end{array}\right]\right\rangle_{\mathcal{H}\left(\widehat{K}_{S}\right) \oplus \mathcal{U}} .
\end{gathered}
$$

Since these inner products match up, the map $V$ defined by

$$
V:\left[\begin{array}{c}
\bar{w} \mathbf{e}_{1}(w)^{*} y+\mathbf{e}_{2}(w)^{*} u \\
y+S(\bar{w}) u
\end{array}\right] \mapsto\left[\begin{array}{c}
\mathbf{e}_{1}(w)^{*} y+\bar{w} \mathbf{e}_{2}(w)^{*} u \\
S(w)^{*} y+u
\end{array}\right]
$$

extends by linearity and continuity to an isometry (also denoted by $V$ ) from

$\mathcal{D}=\overline{\operatorname{span}}\left\{\left[\begin{array}{c}\bar{w} \mathbf{e}_{1}(w)^{*} y+\mathbf{e}_{2}(w)^{*} u \\ y+S(\bar{w}) u\end{array}\right]: u \in \mathcal{U}, y \in \mathcal{Y}, w \in \mathbb{D}\right\} \subset\left[\begin{array}{c}\mathcal{H}\left(\widehat{K}_{S}\right) \\ \mathcal{Y}\end{array}\right]$

onto

$\mathcal{R}:=\overline{\operatorname{span}}\left\{\left[\begin{array}{c}\mathbf{e}_{1}(w)^{*} y+\bar{w} \mathbf{e}_{2}(w)^{*} u \\ S(w)^{*} y+u\end{array}\right]: u \in \mathcal{U}, y \in \mathcal{Y}, w \in \mathbb{D}\right\} \subset\left[\begin{array}{c}\mathcal{H}\left(\widehat{K}_{S}\right) \\ \mathcal{U}\end{array}\right]$.

By taking $y=0$ and $w=0$ in the expression for the generic element of $\mathcal{D}$, one can see that $\mathcal{D} \supset\left[\begin{array}{c}\{0\} \\ \mathcal{Y}\end{array}\right]$. As $u \in \mathcal{U}$ and $y \in \mathcal{Y}$ are independent of 
each other, it follows that the projection down to the first component contains all the kernel functions

$$
\widehat{K}(\cdot, w)\left[\begin{array}{l}
y \\
u
\end{array}\right]=\mathbf{e}_{1}(w)^{*} y+\mathbf{e}_{2}(w)^{*} u
$$

and hence $\mathcal{D}$ in fact is all of $\mathcal{H}(\widehat{K}) \oplus \mathcal{Y}$. Similarly one can see that $\mathcal{R}$ is all of $\mathcal{H}(\widehat{K}) \oplus \mathcal{U}$, and hence $V$ in fact is a unitary transformation from $\mathcal{H}\left(\widehat{K}_{S}\right) \oplus \mathcal{Y}$ onto $\mathcal{H}\left(\widehat{K}_{S}\right) \oplus \mathcal{U}$. From (4.3) it follows that

$$
\left[\begin{array}{ll}
A^{*} & C^{*} \\
B^{*} & D^{*}
\end{array}\right]\left[\begin{array}{c}
\bar{w} \mathbf{e}_{1}(w)^{*} y+\mathbf{e}_{2}(w)^{*} u \\
y+S(\bar{w}) u
\end{array}\right]=\left[\begin{array}{c}
\mathbf{e}_{1}(w)^{*} y+\bar{w} \mathbf{e}_{2}(w)^{*} u \\
S(w)^{*} y+u
\end{array}\right] .
$$

As $V$ is actually unitary, it also follows that

$$
\left[\begin{array}{cc}
A & B \\
C & D
\end{array}\right]\left[\begin{array}{c}
\mathbf{e}_{1}(w)^{*} y+\bar{w} \mathbf{e}_{2}(w)^{*} u \\
S(w)^{*} y+u
\end{array}\right]=\left[\begin{array}{c}
\bar{w} \mathbf{e}_{1}(w)^{*} y+\mathbf{e}_{2}(w)^{*} u \\
y+S(\bar{w}) u
\end{array}\right] .
$$

Upon setting $u=0$ in (4.4), one arrives at

$$
\left[\begin{array}{ll}
A^{*} & C^{*} \\
B^{*} & D^{*}
\end{array}\right]\left[\begin{array}{c}
\bar{w} \mathbf{e}_{1}(w)^{*} y \\
y
\end{array}\right]=\left[\begin{array}{c}
\mathbf{e}_{1}(w)^{*} y \\
S(w)^{*} y
\end{array}\right] .
$$

The next step is to proceed as was done in the proof of $(1) \Rightarrow$ (4) in Theorem 2.1 to get

$$
\mathbf{e}_{1}(w)^{*} y=\left(I-\bar{w} A^{*}\right)^{-1} C^{*} y
$$

from the first row of (4.4) and then use this in the second row to get

$$
\bar{w} B^{*}\left(I-\bar{w} A^{*}\right)^{-1} C^{*} y+D^{*} y=S(w)^{*} y .
$$

Then taking adjoints and setting $z=\bar{w}$ leads to the unitary realization for $S$ :

$$
S(z)=D+z C(I-z A)^{-1} B .
$$

Alternatively, one may set $y=0$ in (4.5) to get

$$
\left[\begin{array}{cc}
A & B \\
C & D
\end{array}\right]\left[\begin{array}{c}
\bar{w} \mathbf{e}_{2}(w)^{*} u \\
u
\end{array}\right]=\left[\begin{array}{c}
\mathbf{e}_{2}(w)^{*} u \\
S(\bar{w}) u
\end{array}\right]
$$

The first row can be solved for $\mathbf{e}_{2}(w)^{*} u$ to get

$$
\mathbf{e}_{2}(w)^{*} u=(I-\bar{w} A)^{-1} B u .
$$

From the second row it then follows that

$$
\bar{w} C(I-\bar{w} A)^{-1} B u+D u=S(\bar{w}) u .
$$

Letting $z=\bar{w} \in \mathbb{D}$ then again leads to the realization (4.7). As $V$ (and $V^{*}$ ) is unitary, either way leads to a unitary realization (2.15) for $S$. It remains to identify $V^{*}=\left[\begin{array}{cc}A & B \\ C & D\end{array}\right]$ with the model colligation $\widehat{\mathbf{U}}_{S}=\left[\begin{array}{ll}\widehat{A}_{S} & \widehat{B}_{S} \\ \widehat{C}_{S} & \widehat{D}_{S}\end{array}\right]$ given by (2.14). 
From (4.6) with $w=0$ it is seen that $C^{*} y=\mathbf{e}_{1}(0)^{*} y$. A simple adjoint computation now gives

$$
C:\left[\begin{array}{l}
f \\
g
\end{array}\right] \rightarrow f(0)
$$

so $C=\widehat{C}_{S}$. With the action of $C^{*}$ identified, one can use the first row of (4.6) to solve for $A^{*} \mathbf{e}_{1}(w)^{*} y$ :

$$
A^{*} \mathbf{e}_{1}(w)^{*} y=\frac{\mathbf{e}_{1}(w)^{*}-\mathbf{e}_{1}(0)^{*}}{\bar{w}} y .
$$

Then a simple adjoint computation

$$
\left\langle A\left[\begin{array}{l}
f \\
g
\end{array}\right], \mathbf{e}_{1}(w)^{*} y\right\rangle_{\mathcal{H}(\widehat{K})}=\left\langle\left[\begin{array}{l}
f \\
g
\end{array}\right], \frac{\mathbf{e}_{1}(w)^{*}-\mathbf{e}_{1}(0)^{*}}{\bar{w}} y\right\rangle_{\mathcal{H}(\widehat{K})}=\left\langle\frac{f(w)-f(0)}{w}, y\right\rangle_{\mathcal{Y}}
$$

reveals what the action of $A$ is in the first component. From (4.4) with $y=0$ gives

$$
A^{*} \mathbf{e}_{2}(w)^{*} u+C^{*} S(\bar{w}) u=\bar{w} \mathbf{e}_{2}(w)^{*} y
$$

or

$$
A^{*} e_{2}(w)^{*} u=\bar{w} \mathbf{e}_{2}(w)^{*} u-\mathbf{e}_{1}(0)^{*} S(\bar{w}) u .
$$

Thus

$$
\begin{aligned}
\left\langle A\left[\begin{array}{l}
f \\
g
\end{array}\right], \mathbf{e}_{2}(w)^{*} u\right\rangle_{\mathcal{H}(\widehat{K})} & =\left\langle\left[\begin{array}{l}
f \\
g
\end{array}\right], A^{*} \mathbf{e}_{2}(w)^{*} u\right\rangle_{\mathcal{H}(\widehat{K})} \\
& =\left\langle\left[\begin{array}{l}
f \\
g
\end{array}\right], \bar{w} \mathbf{e}_{2}(w)^{*} u-\mathbf{e}_{1}(0)^{*} S(\bar{w}) u\right\rangle_{\mathcal{H}(\widehat{K})} \\
& =\left\langle w g(w)-S(\bar{w})^{*} f(0), u\right\rangle_{\mathcal{U}}
\end{aligned}
$$

This completes the confirmation that $A=\widehat{R}_{0}$.

From (4.5) with $y=0$ and $w=0$ one sees that

$$
B u=e_{2}(0)^{*} u=\left[\begin{array}{c}
([S(z)-S(0)] / z) u \\
K_{\widetilde{S}}(z, w) u
\end{array}\right]=\widehat{B}_{S} u .
$$

Finally, setting $w=0$ in (4.8) gives $D=S(0)$. This completes the verification that $V^{*}=\widehat{R}_{0}$. The verification that $V=\left(\widehat{R}_{0}\right)^{*}$ is given by (2.12) is symmetric (interchange the roles of the components $\left[\begin{array}{l}f \\ g\end{array}\right]$ in an element of $\mathcal{D}(S)$ ). The norm identities (2.13) are simple consequences of $V$ being unitary. This completes the verification of $(1) \Rightarrow(2) \&(3)$, (4) in Theorem 2.2

The following verification of $(1) \Rightarrow(5)$ in Theorem 2.2 very much parallels the proof in Section 3.4 for the corresponding result in Theorem 2.1. 
Proof of $(1) \Rightarrow(5)$ in Theorem 2.2. Let $A$ be a completely nonunitary contraction operator on the Hilbert space $\mathcal{X}$. Let $\mathbf{U}=\left[\begin{array}{ll}A & B \\ C & D\end{array}\right]$ be a Julia operator for $A$ (see [DR1991]), also known as a Halmos dilation of $A$ (see [Ha1950])); by this is meant that $B, C, D$ are constructed so that $\mathbf{U}$ is unitary with $B$ injective and with $C$ having dense range. The simplest choice of $B, C, D$ is to take $\mathcal{U}=\mathcal{D}_{A^{*}}=\overline{\operatorname{Ran}} D_{A^{*}}$ where $D_{A^{*}}=\left(I-A A^{*}\right)^{\frac{1}{2}}, \mathcal{Y}=\mathcal{D}_{A}=\overline{\operatorname{Ran}} D_{A}$ where $D_{A}=\left(I-A^{*} A\right)^{\frac{1}{2}}$ and set

$$
\mathbf{U}=\left.\left[\begin{array}{cc}
A & D_{A^{*}} \\
D_{A} & -A^{*}
\end{array}\right]\right|_{\mathcal{X} \oplus \mathcal{D}_{A^{*}}}:\left[\begin{array}{c}
\mathcal{X} \\
\mathcal{D}_{A^{*}}
\end{array}\right] \rightarrow\left[\begin{array}{c}
\mathcal{X} \\
\mathcal{D}_{A}
\end{array}\right]
$$

The next step is to define a map $\Xi: \mathcal{X} \rightarrow\left[\begin{array}{l}H^{2}(\mathcal{Y}) \\ H^{2}(\mathcal{U})\end{array}\right]$ by

$$
\Xi: x \mapsto\left[\begin{array}{c}
\mathcal{O}_{C, A} x \\
\mathcal{O}_{B^{*}, A^{*}} x
\end{array}\right]=\left[\begin{array}{c}
C(I-z A)^{-1} x \\
B^{*}\left(I-z A^{*}\right)^{-1} x
\end{array}\right] .
$$

The assumption that $A$ is completely nonunitary guarantees that $\Xi$ is injective. Let $\mathcal{D}_{0}$ be the range of the map $\Xi$ with the pullback norm:

$$
\|\Xi x\|_{\mathcal{D}_{0}}=\|x\|_{\mathcal{X}} .
$$

Just as in the proof done in Section 3.4 for $(1) \Rightarrow(5)$ in Theorem 2.1, one can verify that $\mathcal{D}_{0}$ is a reproducing kernel Hilbert space with reproducing kernel $K_{C, A, B}$ given by

$$
\left.K_{C, A, B}(z, w)=\left[\begin{array}{c}
C(I-z A)^{-1} \\
B^{*}\left(I-z A^{*}\right)^{-1}
\end{array}\right]\left[I-\bar{w} A^{*}\right)^{-1} C^{*} \quad(I-\bar{w} A)^{-1} B\right] .
$$

Set $S(z)=D+z C(I-z A)^{-1} B$; then $S$ is in the Schur class $\mathcal{S}(\mathcal{U}, \mathcal{Y})$ and, as was already observed above (see (4.1) ), the fact that $\mathbf{U}=\left[\begin{array}{ll}A & B \\ C & D\end{array}\right]$ is unitary implies that $K_{C, A, B}(z, w)=\widehat{K}_{S}(z, w)$. It is now a straightforward (if perhaps tedious) exercise to verify that $\widehat{R}_{0} \Xi=\Xi A$. As $\Xi: \mathcal{X} \rightarrow \mathcal{D}_{0}=\mathcal{D}(S)$ is unitary, it follows that $A$ is unitarily equivalent to the operator $\widehat{R}_{0}$ on $\mathcal{D}(S)$.

Remark 4.1. Those familiar with the Sz.-Nagy-Foias model theory [SNF1970] will notice that the function $S$ produced in the preceding proof is just the Sz.-Nagy-Foias characteristic function for the operator $A^{*}$.

4.2. $\mathcal{D}(S)$ as a pullback space. By definition, the two-component de Branges-Rovnyak space $\mathcal{D}(S)$ sits inside the direct sum Hardy space $H^{2}(\mathcal{Y}) \oplus H^{2}(\mathcal{U})$. Nikolskii and Vasyunin (see [NV1989, NV1998]) have introduced an adjusted version which sits inside $H^{2}(\mathcal{Y}) \oplus\left(L^{2}(\mathcal{U}) \ominus H^{2}(\mathcal{U})\right)$ which is a more natural object to look at in the context of Sz.-Nagy dilation theory and Lax-Phillips scattering as will be now described. 
The adjustment is simple enough: note that the map $j: f(\zeta) \mapsto$ $\bar{\zeta} f(\bar{\zeta})$ is an involution on $L^{2}(\mathbb{T}, \mathcal{U})$ which transforms $H^{2}(\mathcal{U})$ to $H^{2}(\mathcal{U})^{\perp}:=$ $L^{2}(\mathcal{U}) \ominus H^{2}(\mathcal{U})$ and vice versa. Use the notation $\widetilde{D}(S)$ for the flip of the space $\mathcal{D}(S)$ defined by

$$
\widetilde{\mathcal{D}}(S)=\left\{\left[\begin{array}{l}
f \\
g
\end{array}\right]:\left[\begin{array}{c}
f \\
j(g)
\end{array}\right] \in \mathcal{D}(S)\right\} .
$$

Then it is shown in NV1989] that $\widetilde{\mathcal{D}}(S)$ can be identified with the pullback space

$$
\widetilde{\mathcal{D}}(S)=\left.\operatorname{Ran}\left[\begin{array}{cc}
I_{H^{2}(\mathcal{Y})} & L_{S} \\
L_{S}^{*} & I_{H^{2}(\mathcal{U})^{\perp}}
\end{array}\right]\right|_{H^{2}(\mathcal{Y}) \oplus H^{2}(\mathcal{U})^{\perp}}
$$

with the pullback norm

$\left\|\left[\begin{array}{cr}I & S \\ S^{*} & I\end{array}\right]\left[\begin{array}{l}f \\ g\end{array}\right]\right\|_{\widetilde{\mathcal{D}}(S)}=\left\|\mathbf{Q}\left[\begin{array}{l}f \\ g\end{array}\right]\right\|_{H^{2}(\mathcal{Y}) \oplus H^{2}(\mathcal{U})^{\perp}}$, where $\mathbf{Q}=P\left(\operatorname{Ker}\left[\begin{array}{cc}I & L_{S} \\ L_{S}^{*} & I\end{array}\right]\right)^{\perp,}$

or, in the notation of Section 3.2 ,

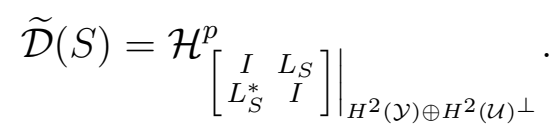

The utility of this formulation is that one can see better the unitary dilation space and the unitary dilation of the model operator $\widehat{R}_{0}$ as well as the associated Lax-Phillips scattering. Recall that, for $T$ a contraction operator on $\mathcal{H}$, by a theorem of Sz.-Nagy (see SNF1970, D1974), $T$ has a unitary dilation $U$, i.e., there is a unitary operator $U$ on a Hilbert space $\mathcal{K} \supset \mathcal{H}$ such that

$$
T^{n}=\left.P_{\mathcal{H}} U^{n}\right|_{\mathcal{H}} \quad \text { for } \quad n=0,1,2, \ldots
$$

By Sarason's lemma [S1965], the space $\mathcal{K}$ has a decomposition

$$
\mathcal{K}=\mathcal{G}_{*} \oplus \mathcal{H} \oplus \mathcal{G} \quad \text { where } \quad U^{*} \mathcal{G}_{*} \subset \mathcal{G}_{*}, \quad U \mathcal{G} \subset \mathcal{G} .
$$

When the unitary dilation $U$ is minimal, i.e., when $\mathcal{K}=\overline{\operatorname{span}}\left\{U^{n} \mathcal{H}: n \in\right.$ $\mathbb{Z}\}$, then necessarily $\left.U^{*}\right|_{\mathcal{G}_{*}}$ and $\left.U\right|_{\mathcal{G}}$ are pure isometries, and hence

$$
\mathcal{G}_{*}=\bigoplus_{n \geq 0} U^{* n}\left(\mathcal{G}_{*} \ominus U^{*} \mathcal{G}_{*}\right), \quad \mathcal{G}=\bigoplus_{n \geq 0} U^{n}(\mathcal{G} \ominus U \mathcal{G})
$$

When the completely nonunitary contraction operator $T$ is modeled as the model operator

$$
\left(\widetilde{\widehat{R}_{0}}\right)^{*}:\left[\begin{array}{c}
f(\zeta) \\
g(\zeta)
\end{array}\right] \mapsto\left[\begin{array}{c}
\zeta f(\zeta)-S(\zeta)[g]_{-1} \\
\zeta g(\zeta)-[g]_{-1}
\end{array}\right]
$$


on $\widetilde{\mathcal{D}}(S)$ (where now the functions in the model space are written as functions of the variable $\zeta$ on the circle $\mathbb{T})$, then it can be shown that the unitary dilation for $T=\left(\widetilde{R}_{0}\right)^{*}$ can be modeled as the operator

$$
M_{\zeta}:\left[\begin{array}{l}
f(\zeta) \\
g(\zeta)
\end{array}\right] \mapsto\left[\begin{array}{l}
\zeta f(\zeta) \\
\zeta g(\zeta)
\end{array}\right]
$$

on the pullback space

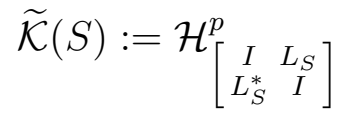

where the operator $\left[\begin{array}{cc}I & L_{S} \\ L_{S}^{*} & I\end{array}\right]$ is now viewed as acting of $L^{2}(\mathcal{Y}) \oplus L^{2}(\mathcal{U})$. The decomposition (4.10) is valid with $\mathcal{K}=\widetilde{\mathcal{K}}(S)$ as in (4.11), $\mathcal{H}=$ $\widetilde{\mathcal{D}}(S)$ as in (4.9), and with the incoming space $\mathcal{G}_{*}=\widetilde{\mathcal{G}}_{*}(S)$ and the outgoing space $\mathcal{G}=\widetilde{\mathcal{G}}(S)$ given respectively by

$$
\widetilde{\mathcal{G}}_{*}(S)=\mathcal{H}_{\left[\begin{array}{c}
I \\
L_{S}^{*}
\end{array}\right] \mid H^{2}(\mathcal{Y})^{\perp}} \quad \text { and } \quad \widetilde{\mathcal{G}}(S)=\mathcal{H}_{\left[\begin{array}{c}
L_{S} \\
I
\end{array}\right] \mid H^{2}(\mathcal{Y})}^{p} .
$$

In the Nikolskii-Vasyunin terminology, there is a coordinate-free formulation of the model for a completely nonunitary contraction and the associated unitary dilation, and this de Branges-Rovnyak formulation is but one of three possible transcriptions, the others being the Sz.-Nagy-Foias and the Pavlov transcriptions. A thorough extension of all these ideas to multievolution scattering systems and Schur-class functions on the polydisk (rather than on the unit disk), where the realization theory has man more subtleties and complications, is carried out in BSV2005.

\subsection{Two-component overlapping spaces: factorization and in-} variant subspaces. The following enhanced generalization of Proposition 3.18 is relevant to these issues.

Theorem 4.2. (See [BB1970, B1978, BC1991]). Let $\left.S_{1} \in \mathcal{S}\right)\left(\mathcal{U}_{0}, \mathcal{U}\right)$ and $S_{2} \in \mathcal{S}(\mathcal{U}, \mathcal{Y})$ be two Schur-class functions for which the product $S=S_{2} \cdot S_{1} \in \mathcal{S}\left(\mathcal{U}_{0}, \mathcal{Y}\right)$ is defined. Then the map

$$
Z:\left[\begin{array}{l}
f_{2} \\
g_{2}
\end{array}\right] \oplus\left[\begin{array}{l}
f_{1} \\
g_{1}
\end{array}\right] \mapsto\left[\begin{array}{l}
f_{2}(z)+S_{2}(z) f_{1}(z) \\
\widetilde{S}_{1}(z) g_{2}(z)+g_{1}(z)
\end{array}\right]
$$

is a coisometry from $\mathcal{D}\left(S_{2}\right) \bigoplus \mathcal{D}\left(S_{1}\right)$ onto $\mathcal{D}\left(S_{2} S_{1}\right)$. If one defines the two-component overlapping space $\mathcal{E}\left(S_{2} \cdot S_{1}\right)$ by

$$
\mathcal{E}\left(S_{2} \cdot S_{1}\right)=\left\{\left[\begin{array}{l}
f \\
g
\end{array}\right] \in H^{2}(\mathcal{U}):\left[\begin{array}{c}
S_{2} f \\
-g
\end{array}\right] \in \mathcal{D}\left(S_{2}\right) \text { and }\left[\begin{array}{c}
-f \\
\widetilde{S}_{1} g
\end{array}\right] \in \mathcal{D}\left(S_{1}\right)\right\}
$$


with norm

$$
\left\|\left[\begin{array}{l}
f \\
g
\end{array}\right]\right\|^{2}=\left\|\left[\begin{array}{c}
S_{2} f \\
-g
\end{array}\right]\right\|^{2}+\left\|\left[\begin{array}{c}
-f \\
\widetilde{S}_{1} g
\end{array}\right]\right\|^{2},
$$

then the map

$$
\chi:\left[\begin{array}{l}
f \\
g
\end{array}\right] \mapsto\left[\begin{array}{l}
S_{2} f \\
-g
\end{array}\right] \oplus\left[\begin{array}{l}
-f \\
\widetilde{S}_{1} g
\end{array}\right]
$$

is a unitary embedding of $\mathcal{E}\left(S_{2} \cdot S_{1}\right)$ into Ker $Z$. Furthermore, $\mathcal{E}\left(S_{2} \cdot S_{1}\right)$ is RKHS with reproducing kernel $K_{S_{2} \cdot S_{1}}$ given by

$K_{S_{2} \cdot S_{1}}(z, w)=\left[\begin{array}{cc}\frac{1}{2}(1-z \bar{w})^{-1}\left(\varphi(z)+\varphi(w)^{*}\right) & \frac{1}{2}(z-\bar{w})^{-1}(\varphi(z)-\varphi(\bar{w})) \\ \frac{1}{2}(z-\bar{w})^{-1}(\widetilde{\varphi}(z)-\widetilde{\varphi}(\bar{w}) & \frac{1}{2}(1-z \bar{w})^{-1}\left(\widetilde{\varphi}(z)+\widetilde{\varphi}(w)^{*}\right)\end{array}\right]$

where in this case $\varphi$ is given by

$$
\varphi(z)=\int_{\mathbb{T}} \frac{\zeta+z}{\zeta-z} \Omega(\zeta) \frac{|\mathrm{d} \zeta|}{2 \pi}
$$

and $\Omega(\zeta)$ is a certain positive semidefinite operator on $\mathbb{T}$ determined by the two pointwise defect operators $\Omega_{2}(\zeta):=I-S_{2}(\zeta)^{*} S_{2}(\zeta)$ and $\Omega_{1}(\zeta):=I-S_{1}(\zeta) S_{1}(\zeta)^{*}$; when $\Omega_{2}(\zeta)$ and $\Omega_{1}(\zeta)$ are both invertible, then $\Omega(\zeta)$ is determined from the identity

$$
\Omega(\zeta)^{-1}=\Omega_{2}(\zeta)^{-1}+\Omega_{1}(\zeta)^{-1}-I
$$

Remark 4.3. It turns out that Theorem 4.2 is connected with factorization and invariant subspaces. A sketch of the explanation with getting into precise details is as follows. As explained in Brodskii [Br1978] and Ball-Cohen [BC1991], invariant subspaces for the state operator $\widehat{R}_{0}^{*}$ on $\mathcal{D}(S)$ are determined by nontrivial regular factorizations $S=S_{2} \cdot S_{1}$ of the characteristic function $S$. The factorization $S=S_{2} \cdot S_{1}$ being regular corresponds to the overlapping space $\mathcal{E}\left(S_{2} \cdot S_{1}\right)$ being trivial, or to $Z$ being unitary from $\mathcal{D}\left(S_{2}\right) \oplus \mathcal{D}\left(S_{1}\right)$ to $\mathcal{D}(S)$ (see [B1978] and [BC1991, Section 7]), or to $Z$ being unitary from $\mathcal{D}\left(S_{2}\right) \oplus \mathcal{D}\left(S_{1}\right)$ to $\mathcal{D}(S)$. Tracing through the form of the operator $\widehat{R}_{0}^{*}$ on $\mathcal{D}(S)$ in the alternative decomposition $\mathcal{D}\left(S_{2}\right) \oplus \mathcal{D}\left(S_{1}\right)$ obtained by applying $Z^{*}$ to $\mathcal{D}(S)$, one can see that $0 \oplus \mathcal{D}\left(S_{1}\right)$ is an invariant subspace of $\widehat{R}_{0}^{*}$; by the general theory of cascade decompositions of unitary colligations (see [Br1978] and [BC1991]), every invariant subspace for $\widehat{R}_{0}^{*}$ arises in this way. The problem of finding nontrivial invariant subspaces for a completely nonunitary contraction operator is therefore transferred to the problem of finding nontrivial regular factorizations for characteristic operator functions; as these Schur-class functions in general act between infinite-dimensional coefficient Hilbert spaces, this problem in turn is tractable only with additional assumptions. it is a curious fact, 
nonetheless, that even when the factorization is not regular, one still gets an invariant subspace, but for $\widehat{R}_{0}^{*} \oplus U$ on $\mathcal{D}(S) \oplus \mathcal{E}\left(S_{2} \cdot S_{1}\right)$ rather than for $\widehat{R}_{0}^{*}$ itself; here $U$ is the unitary operator on $\mathcal{E}\left(S_{2} \cdot S_{1}\right)$ given by

$$
U:\left[\begin{array}{l}
f(z) \\
g(z)
\end{array}\right] \mapsto\left[\begin{array}{c}
z f(z)-g(0) \\
{[g(z)-g(0)] / z}
\end{array}\right] .
$$

This result was also obtained independently in the setting of the Sz.Nagy-Foias model (see [SNF1970, Notes to Chapter VII]). While this phenomenon appears to be disappointing from the point of view of searching for invariant subspaces, it is exactly the tool used in [B1978] to obtain the spectral invariants for the unitary part of a whole class of contractive integral operators defined on a vector-valued $L^{2}$-space on the unit circle (see also [K1976] for a real-line version).

Finally, it turns out that the operator $\Omega(\zeta)$ appearing in Theorem 4.2 satisfies

$$
\operatorname{Ran} \Omega(\zeta)^{1 / 2}=\operatorname{Ran} \Omega_{2}(\zeta)^{1 / 2} \cap \operatorname{Ran} \Omega_{1}(\zeta)^{1 / 2} .
$$

Thus the operator $\Omega(\zeta)$ is related to but not quite the same as the parallel sum of $\Omega_{1}(\zeta)$ and $\Omega_{2}(\zeta)$ studied by Fillmore and Williams FW1971 with motivation from circuit theory; the parallel sum studied in FW1971 also satisfies the range-intersection property (4.14) but is determined in simple cases by the parallel-sum identity

$$
\Omega(\zeta)^{-1}=\Omega_{2}(\zeta)^{-1}+\Omega_{1}(\zeta)^{-1}
$$

rather than by the Brangesian parallel-sum identity (4.13). Nevertheless, a consequence of the range intersection property (4.14) is that the overlapping space $\mathcal{E}\left(S_{2} \cdot S_{1}\right)$ is trivial, i.e., the factorization $S=S_{2} \cdot S_{1}$ is regular, if and only if

$$
\operatorname{Ran} \Omega_{2}(\zeta)^{1 / 2} \cap \operatorname{Ran} \Omega_{1}(\zeta)^{1 / 2}=\{0\} \text { for a.e. } \zeta \in \mathbb{T}
$$

(see [B1978]). An independent direct proof for this factorization-regularity criterion was given in the setting of the Sz.-Nagy-Foias model theory in [SNF1974].

\section{Generalizhtions And extensions}

\subsection{Canonical de Branges-Rovnyak functional-model spaces:} multivariable settings. Realization of a Schur-class function as the transfer function of a canonical functional-model colligation having additional metric properties (e.g., coisometric, isometric, or unitary), i.e., item (4) in Theorems 2.1 an 2.2, has been extended to settings where the unit disk playing the role of the underlying domain is replaced by a more general domain $\mathcal{D}$ in $\mathbb{C}^{d}$; see BB2012c for the case of the 
unit ball $\mathbb{B}^{d}$ in $\mathbb{C}^{d}$, $\mathrm{BB} 2012 \mathrm{~b}$. for the case of the unit polydisk $\mathbb{D}^{d}$, BB2012a for the case of a general domain with matrix polynomial defining function, and [BB2010] for an overview of all three settings. In these multivariable settings, the backward shift operator $R_{0}$ is replaced by a solution of the Gleason problem; an early manifestation of this idea is in ADR2003. For the case where the origin is in the domain $\mathcal{D} \subset \mathbb{C}^{d}$, the Gleason problem (centered at 0 ) can be formulated as: given $f$ in our space of functions $\mathcal{F}$ on $\mathcal{D}$, find $f_{1}, \ldots, f_{d}$ also in $\mathcal{F}$ so that $f(z)=f(0)+\sum_{k=1}^{d} z_{k} f_{k}(z)$. As the solution of such a Gleason problem is often not unique, one speaks about many de Branges-Rovnyak spaces $\mathcal{H}(S)$ or $\mathcal{D}(S)$ associated with a given function $f$ in the generalized Schur-class over the domain $\mathcal{D}$; as long as certain minimal structural components are maintained, all these are called canonical functional models going with the same $S$. One then gets good uniqueness results in the following sense: any other transferfunction realization satisfying certain observability/controllability and weak metric properties is unitarily equivalent to some functional-model transfer-function realization.

There has also been work on extending the functional-model aspect (item (5) in Theorems 2.1 and 2.2), at least in the ball setting, where a commutative row contraction, i.e., a commutative $d$-tuple of operators $T_{1}, \ldots, T_{d}$ on a Hilbert space $\mathcal{H}$ for which the block row $\left[\begin{array}{lll}T_{1} & \cdots & T_{d}\end{array}\right]: \mathcal{H}^{d} \rightarrow \mathcal{H}$ is contractive, replaces a single contraction operator $T$ (see [BES2005, BB2012c]). There has also been extensive work on noncommutative versions (models for a not necessarily commutative operator $d$-tuple with block-row matrix $\left[\begin{array}{lll}T_{1} & \cdots & T_{d}\end{array}\right]$ contractive - see [Bu1984, F1982, P1989a, P1989b, P1995, BV2005, BBF2007b]) which then leads into noncommutative function theory. For lack of space, these matters are not dealt with in any detail here.

5.2. Extensions to Kreĭn space settings. Much of the theory of de Branges-Rovnyak spaces given in Sections 3 and 4 actually extends to Pontryagin and Kreın-space settings, where Hilbert spaces coming up in various places are allowed to be Krĕn spaces (i.e., the space is a direct sum of a Hilbert space and an anti-Hilbert space), or at least Pontryagin spaces (where the anti-Hilbert space is finite dimensional). In particular, the paper of de Branges dB1988 provides a nice extension of the theory of complementary spaces developed in Section 3 above to the Kreln-space setting; the book [ADRS1997], besides reviewing these matters, also develops the whole realization theorem (item (4) in Theorems 2.1 and 2.2) to the Pontryagin-space setting (see also [DR1991]). One place where these generalizations are relevant is 
in the proof of the Bieberbach conjecture. Certain relevant inequalities involve contraction operators on a Pontryagin function space, involving substitution (or composition) contraction operators $T$ rather than multiplication contraction operators $T=T_{S}$ associated with a Schur function $S$ (see [dB1984, dB1985]).

The Pontryagin-space formulation of the Nikolskii-Vasyunin model space $\widetilde{\mathcal{D}}(S)$ in terms of Krein-Langer representations was given in [De2001, De2003] as a necessary step to formulate a general interpolation problem for generalized Schur functions.

\section{Concluding Remarks}

The preceding sections give an overview of the basic properties of de Branges Rovnyak spaces along with their applications to related function theory and operator theory problems. It is worth noting that the theory and applications are still evolving, as illustrated by the following examples.

6.1. Still other settings. Whenever one has a substitute for the Hardy space $H^{2}(\mathcal{Y})$ and of the Schur class $\mathcal{S}(\mathcal{U}, \mathcal{Y})$, possibly in its role as the multipliers from $H^{2}(\mathcal{U})$ to $H^{2}(\mathcal{Y})$, one has a notion of de Branges-Rovnyak space $\mathcal{H}(S)$. An easier first case is the case where $S$ is inner, so that $\mathcal{H}(S)=H^{2}(\mathcal{Y}) \ominus S \cdot H^{2}(\mathcal{U})$ is just a Hardy-space orthogonal difference. Just as in the multivariable context mentioned in Section 5.1 above, one of the issues often is to find the appropriate substitute or analog for the difference-quotient transformation $R_{0}: f(z) \mapsto[f(z)-f(0)] / z$. These ideas have been explored at least in a preliminary way in the following situations:

(1) Quaternionic settings: Two distinct flavors of this topic are slice hyperholomorphic functions [ACS2012, and Fueterregular functions ASV2004.

(2) Riemann-surface settings: See [AV2002, BV2001.

(3) Subbergman spaces: See [Z1996, Z2003] and [BB2013] for the treatment of an interpolation problem in sub-Bergman spaces.

(4) de Branges-Rovnyak spaces over a half plane: The paper of Ball-Kurula-Staffans-Zwart [BKSZ2013] extends the canonical de Branges-Rovnyak functional-model colligation to the right half plane setting and thereby gets canonical-model energypreserving and co-energy-preserving system realizations for Schurclass functions over the right half plane. Fricain and Mashreghi Fri2008 studied the boundary behavior of derivatives of functions in a de Branges-Rovnyak space over the upper half plane. 
6.2. Special questions. Researchers have used de Branges-Rovnyak spaces as a tool to treat various types of special questions. Examples are:

(1) Riesz bases of reproducing kernels: Given $S \in \mathcal{S}(\mathcal{U}, \mathcal{Y})$, $\left\{z_{n}\right\} \subset \mathbb{D}$ and $\left\{y_{n}\right\} \subset \mathcal{Y}$, find a criterion for $\left\{K_{S}\left(\cdot, z_{n}\right) u_{n}\right\}$ to a Riesz basis for $\mathcal{H}(S)$. A reference for this topic is [CFT2009].

(2) Multiplication by finite Blaschke products: On which spaces algebraically included in $H^{2}$ does multiplication by a finite Blaschke product act as an isometry? See [ST1997.

6.3. Applications. De Branges-Rovnyak spaces appear naturally in the context of Schur-class interpolation theory; the prominent role played by these spaces in interpolation theory is discussed in detail in separate survey [BB2014]. Besides the $H^{\infty}$-interpolation theory de Branges-Rovnyak spaces have also appeared as a useful tool in a number of other applications.

(1) Multipliers of de Branges-Rovnyak spaces: References include [C1994, DMcC1991, L1990, LS1991, LS1993, LS1998, Su1995. Interpolation with operator argument is embedded into the scheme of [Bo2003]. All solutions are characterized in terms of positive kernels but there is no more detailed parametrization of the solution set at this level of generality. Some attempts to get realizations for contractive multipliers were done in [AB1997].

(2) Norms of weighted composition operators: See [J2007].

(3) Relative angular derivatives: See [Sha2001, Sha2003]

\section{REFERENCES}

[AMcC2002] J. Agler and J.E. McCarthy, Pick Interpolation and Hilbert Function Spaces, Graduate Studies in Mathematics 44, Amer. Math. Soc., Providence, 2002.

[AB1997] D. Alpay and V. Bolotnikov, On tangential interpolation in reproducing kernel Hilbert modules and applications, in: Topics in Interpolation Theory (Ed. H. Dym et al), pp. 37-68, Oper. Theory Adv. Appl. 95, Birkhäuser, Basel, 1997.

[ACS2012] D. Alpay, F. Colombo and I. Sabadini, Schur functions and their realizations in the slice hyperholomorphic setting, Integral Equations Operator Theory 72 (2012), no. 2, 253-289.

[ADR2003] D. Alpay, A. Dijksma, and J. Rovnyak, A theorem of Beurling-Lax type for Hilbert spaces of functions analytic in the unit ball, Integral Equations Operator Theory 47 (2003) no. 3, 251-274.

[ADRS1997] D. Alpay, A. Dijksma, J. Rovnyak, and H. de Snoo, Schur Functions, Operator Colligations, and Reproducing Kernel Pontryagin Spaces, Oper. Theory Adv. Appl. 96 Birkhäuser (Basel), 1997. 
[ASV2004] D. Alpay, M. Shapiro and D. Volok, Espaces de Branges Rovnyak et fonctions de Schur: le cas hyper-analytique, C. R. Math. Acad. Sci. Paris 338 (2004), no. 6, 437-442.

[AV2002] D. Alpay and V. Vinnikov, Finite dimensional de Branges spaces on Riemann surfaces, J. Funct. Anal. 189 (2002), no. 2, 283-324.

[A1990] T. Ando, De Branges Spaces and Analytic Operator Functions, Hokkaido University (Sapporo), 1990.

[Ar1950] N. Aronszajn, Theory of reproducing kernels, Trans. Amer. Math. Soc., 68 (1950), 337-404.

[AD2008] D. Z. Arov and H. Dym, J-Contractive Matrix Valued Functions and Related Topics, Encyclopedia of Mathematics and its Applications 116, Cambridge University Press, Cambridge, 2008.

[AD2012] D. Z. Arov and H. Dym, Bitangential Direct and Inverse Problems for Systems of Integral and Differential Equations, Encyclopedia of Mathematics and its Applications 145, Cambridge University Press, Cambridge, 2012.

[B1978] J. A. Ball, Factorization and model theory for contraction operators with unitary part, Mem. Amer. Math. Soc. 13 (1978), no. 198.

[B2000] J. A. Ball, Linear systems, operator model theory and scattering: multivariable generalizations, in: Operator Theory and its Applications, pp. 151-178, Fields Inst. Commun., 25, Amer. Math. Soc., Providence, RI, 2000.

[BB2010] J. A. Ball and V. Bolotnikov, Canonical de Branges-Rovnyak model transfer-function realization for multivariable Schur-class functions, in Hilbert Spaces of Analytic Functions (Ed. J. Mashreghi, T. Ransford, and K. Seip), pp. 1-38, CRM Proceedings \& Lecture Notes Volume 51, American Mathematical Society, Providence, 2010.

[BB2012a] J. A. Ball and V. Bolotnikov, Canonical transfer-function realization for Schur-Agler-class functions on domains with matrix polynomial defining functions in $\mathbb{C}^{n}$, in: Recent Progress in Operator Theory and Its Applications (Ed. J.A. Ball, R. Curto, S. Grudsky, W. Helton, R. Quiroga-Barranco and N. Vasilevski) pp. 23-55, Oper. Theory Adv. Appl. 220 Birkhäuser, Basel, 2012.

[BB2012b] J. A. Ball and V. Bolotnikov, Canonical transfer-function realization for Schur-Agler-class functions of the polydisk, in: A Panorama of Modern Operator Theory and Related Topics. The Israel Gohberg Memorial Volume (Ed. H. Dym, M.A. Kaashoek, P. Lancaster, H. Langer, and L. Lerer) pp. 75-122, Oper. Theory Adv. Appl. 218 Birkhäuser (Basel), 2012.

[BB2012c] J. A. Ball and V. Bolotnikov, Canonical transfer-function realization for Schur multipliers on the Drury-Arveson space and models for commuting row contractions, Indiana Univ. Math. J. 61 (2012), 665-716.

[BB2013] J. A. Ball and V. Bolotnikov, Interpolation in sub-Bergman spaces, in: Advances in Structured Operator Theory and Related Areas (Eds. M. Kaashoek et al.), pp. 17-40, Oper. Theory Adv. Appl. 237, Birkhäuser, 2013.

[BB2014] J. A. Ball and V. Bolotnikov, de Branges-Rovnyak spaces and normconstraint interpolation, this issue.

[BBF2007a] J. A. Ball, V. Bolotnikov and Q. Fang, Transfer-function realization for multipliers of the Arveson space, J. Math. Anal. Appl. 333 (2007), no. 1, 68-92. 
[BBF2007b] J. A. Ball, V. Bolotnikov and Q. Fang, Schur-class multipliers on the Fock space: de Branges-Rovnyak reproducing kernel spaces and transferfunction realizations, in Operator Theory, Structured Matrices, and Dilations (Ed. M. Bakonyi, A. Gheondea, M. Putinar, and J. Rovnyak), pp. 85-114, Theta Series in Advanced Mathematics 7, Theta, Bucharest, 2007.

[BBF2008] J. A. Ball, V. Bolotnikov and Q. Fang, Schur-class multipliers on the Arveson space: de Branges-Rovnyak reproducing kernel spaces and commutative transfer-function realizations, J. Math. Anal. Appl. 341 (2008), 519-539.

[BC1991] J. A. Ball and N. Cohen, de Branges-Rovnyak operator models and systems theory: a survey, in: Topics in Matrix and Operator Theory (ed. H. Bart, I. Gohberg, and M.A. Kaashoek) pp. 93-126, Oper. Theory Adv. Appl. OT 50, Birkhäuser, Basel, 1991.

[BK1987] J. A. Ball and T. L. Kriete, Operator-valued Nevanlinna-Pick kernels and the functional models for contraction operators, Integral Equations Operator Theory 10 (1987), 17-61.

[BKSZ2013] J. A. Ball, M. Kurula, O. J. Staffans, and H. Zwart, De BrangesRovnyak realizations of operator-valued Schur functions on the complex right half-plane, arXiv:1307.7408v1.

[BSV2005] J. A. Ball, C. Sadosky and V. Vinnikov, Scattering systems with several evolutions and multidimensional input/state/output systems, Integral Equations Operator Theory 52 (2005), 323-393.

[BV2001] J. A. Ball and V. Vinnikov, Hardy spaces on a finite bordered Riemann surface, multivariable operator model theory, and Fourier analysis along a unimodular curve, in: Systems, Approximation, Singular Integral Operators, and Related Topics (Ed. A.A. Borichev and N.K. Nikolskii) pp. 37-56, Oper. Theory Adv. Appl. 129, Birkhäuser, Basel, 2001.

[BV2003] J. A. Ball and V. Vinnikov, Formal reproducing kernel Hilbert spaces: the commutative and noncommutative settings, in Operator Theory, System Theory and Scattering Theory: Multidimensional Generalizations (Ed. D. Alpay and V. Vinnikov), pp. 77-134, Oper. Theory Adv. Appl. 134, Birkhäuser, Basel, 2003.

[BV2005] J. A. Ball and V. Vinnikov, Lax-Phillips scattering and conservative linear systems: a Cuntz-algebra multidimensional setting, Mem. Amer. Math. Soc. 178 (2005), no. 837.

[BeB1984] F. Beatrous and J. Burbea, Positive-definiteness and its applications to interpolation problems for holomorphic functions, Trans. Amer. Math. Soc., 284 (1984), no.1, 247-270.

[BES2005] T. Bhattacharyya, J. Eschmeier and J. Sarkar, Characteristic function of a pure commuting contractive tuple, Integral Equations Operator Theory $\mathbf{5 3}$ (2005), no. 1, 23-32.

[Bo2003] V. Bolotnikov, Interpolation for multipliers on reproducing kernel Hilbert spaces, Proc. Amer. Math. Soc. 131 (2003), no. 5, 1373-1383

[dB1968] L. de Branges, Hilbert spaces of Entire functions, Prentice-Hall, Englewood Cliffs, 1968.

[dB1970] L. de Branges, Factorization and invariant subspaces, J. Math. Anal. Appl. '29 (1970), 163-200.

[dB1977] L. de Branges, Perturbation theory, J. Mat. Anal. Appl. 57 (1977) no. 2, 393-415. 
[dB1984] L. de Branges, Löwner expansions, J. Math. Anal. Appl. 100 (1984), 323-337.

[dB1985] L. de Branges, A proof of the Bieberbach conjecture, Acta Math. 154 (1985) no. 1-2, 137-152.

[dB1988] L. de Branges, Complementation in Kreìn spaces, Trans. Amer. Math. Soc. 305 (1988), 277-291.

[dBR1966a] L. de Branges and J. Rovnyak, Canonical models in quantum scattering theory, in: Perturbation Theory and its Applications in Quantum Mechanics (C. Wilcox, ed.) pp. 295-392, Holt, Rinehart and Winston, New York, 1966.

[dBR1966b] L. de Branges and J. Rovnyak, Square summable power series, Holt, Rinehart and Winston, New York, 1966.

[dBS1968] L. de Branges and L. Shulman, Perturbations of unitary transformations, J. Math. Anal. Appl. 23 (1968), 294-326.

[Bu1984] J. Bunce, Models for n-tuples of noncommuting operators, J. Funct. Anal. 57 (1984), 21-30.

[Br1978] M. S. Brodskii, Unitary operator colligations and their characteristic functions, Russian Math. Surveys 22 (1978), 159-191.

[CFT2009] N. Chevrot, E. Fricain and D. Timotin, On certain Riesz families in vector-valued de Branges-Rovnyak spaces, J. Math. Anal. Appl. 355 (2009), no. $1,110-125$.

[C1994] B. R. Crofoot, Multipliers between invariant subspaces of the backward shift, Pacific J. Math. 166 (1994), no. 2, 225-246.

[DMcC1991] M.'B. Davis and J. E. McCarthy, Multipliers of de Branges spaces, Michigan Math. J. 38 (1991), no. 2, 225-240.

[De2001] V. A. Derkach, On the indefinite abstract interpolation problem, Methods Funct. Anal. Topol. 7 (2001) no. 4, 87-100.

[De2003] V. A. Derkach, On the indefinite Schur-Nevanlinna-Pick interpolation problem, Ukrain. Mat. Zh. 55 (2003) no. 10, 1299-1313.

[D1965] R. G. Douglas, On majorization, factorization, and range inclusion of operators on Hilbert space, Proc. Amer. Math. Soc. 17 (1966), 413-415.

[D1972] R. G. Douglas, On the structure theory of de Branges and Rovnyak, unpublished.

[D1974] R. G. Douglas, Canonical models, in: Topics in Operator Theory (ed. C. Pearcy) pp.161-218, Mathematical Surveys No. 13, Amer. Math. Soc., Providence, 1974.

[DR1991] M. A. Dritschel and J. Rovnyak, Julia operators and complementation in Krĕ̌n spaces, Indiana Univ. Math. J. 40 No. 3 (1991), 886-901.

[Dy1989] H. Dym, J-contractive matrix functions, reproducing kernel Hilbert spaces and interpolation, CBMS Regional Conference Series in Mathematics, 71, Providence, RI, 1989.

[DyMcK1976] H. Dym and H.P. McKean, Gaussian Processes, Function Theory, and the Inverse Spectral Problem, Academic Press, New York, 1976.

[FW1971] P.A. Fillmore and J.P. Williams, On operator ranges, Adv. Math. 7 (1971), 254-281.

[F1982] A.E. Frazho, Models for noncommuting operators, J. Funct. Anal. 48 (1982), 1-11. 
[Fri2008] E. Fricain and J. Mashreghi, Integral representation of the $n$-th derivative in de Branges-Rovnyak spaces and the norm convergence of its reproducing kernel, Ann. Inst. Fourier, Grenoble 58 (2008) no. 6, 2113-2135.

[Ha1950] P.R. Halmos, Normal dilations and extensions of operators, Summa Brasil 2 (1950), 125-134.

[He1964] H. Helson, Lectures on Invariant Subspaces. Academic Press, 1964.

[J2007] M.T. Jury, Reproducing kernels, de Branges-Rovnyak spaces, and norms of weighted composition operators, Proc. Amer. Math. Soc. 135 (2007), no. 11, 3669-3675.

[K1976] T.L. Kriete, Canonical models and the self-adjoint parts of dissipative operators, J. Funct. Anal. 23 (1976), 39-94.

[L1990] B. A. Lotto, Inner multipliers of de Branges's spaces, Integral Equations Operator Theory 13 (1990), no. 2, 216-230.

[LS1991] B.A. Lotto and D. Sarason, Multiplicative structure of de Branges's spaces, Rev. Mat. Iberoamericana 7 (1991), no. 2, 183-220.

[LS1993] B.A. Lotto and D. Sarason, Multipliers of de Branges-Rovnyak spaces, Indiana Univ. Math. J. 42 (1993), 907-920.

[LS1998] B.A. Lotto and D. Sarason, Multipliers of de Branges-Rovnyak spaces. II, in: Harmonic Analysis and Hypergroups, pp. 51-58, Trends Math., Birkhüser Boston, Boston, 1998.

[NV1986] N.K. Nikolskii and V.I. Vasyunin, Notes on two function models, in: The Bieberbach Conjecture: Proceedings of the Symposium on the Occasion of the Proof (ed.. A. Baernstein et al.), pp. 113-141, Mathematical Surveys and Monographs 21, Amer. Math. Soc. Providence, 1986.

[NV1989] N.K. Nikolskii and V.I. Vasyunin, A unified approach to function models, and the transcription problem, in: The Gohberg Anniversary Collection Vol. 2 (Ed. H. Dym et al.), pp. 405-434, OT41 Birkhäuser-Verlag, Basel-Boston, 1989.

[NV1998] N.K. Nikolskii and V.I. Vasyunin, Elements of spectral theory in terms of the free function model Part I: Basic constructions, in: Holomorphic Spaces (Ed. S. Axler, J.E. McCarthy and D. Sarason), pp. 211-302, Mathematical Sciences Research Institute Publications Vol. 33, Cambridge University Press, 1998.

[P1989a] G. Popescu, Models for infinite sequences of noncommuting operators, Acta Sci. Math. 53 (1989), 355-368.

[P1989b] G. Popescu, Isometric dilations for infinite sequences of noncommuting operators, Trans. Amer. MAth. Soc. 316 (1989), 523-536.

[P1995] G. Popescu, Multi-analytic operators on Fock spaces, Math. Ann. 303 (1995), 31-46.

[R1967] M. Rosenblum, University of Virginia Hilbert Space Seminar Notes, 19671968.

[RR1985] M. Rosenblum and J. Rovnyak, Hardy Classes and Operator Theory, Oxford Mathematical Monographs, Oxford University Press, Oxford, 1985.

[S1965] D. Sarason, On spectral sets having connected complement, Acta Sci. Math. (Szeged) 26 (1965), 289-299.

[S1986] D. Sarason, Shift-invariant spaces from the Brangesian point of view, in: The Bieberbach Conjecture: Proceedings of the Symposium on the Occasion 
of the Proof (Ed. A. Baernstein et al.),, pp. 153-166, Mathematical Surveys and Monographs 21, Amer. Math. Soc. Providence, 1986.

[S1994] D. Sarason, Sub-Hardy Hilbert Spaces in the Unit Disk, John Wiley and Sons Inc., New York, 1994.

[Sha2001] J. E. Shapiro, Relative angular derivatives, J. Operator Theory 46 (2001), no. 2, 265-280.

[Sha2003] J. E. Shapiro, More relative angular derivatives, J. Operator Theory 49 (2003), no. 1, 85-97.

[ST1997] D. Singh and V. Thukral, Multiplication by finite Blaschke factors on de Branges spaces, J. Operator Theory 37 (1997), no. 2, 223-245.

[Su1995] F.D. Suárez, Multipliers of de Branges-Rovnyak spaces in $H^{2}$, Rev. Mat. Iberoamericana 11 (1995), no. 2, 375-415.

[SNF1970] B. Sz.-Nagy, C. Foias, H. Bercovici, and L. Kérchy, Harmonic Analysis of Operators on Hilbert Space: Second Edition, Universitext, Springer, New York, 2010; enlarged and revised edition of first edition by B. Sz.-Nagy and C. Foias, North-Holland, New York, 1970.

[SNF1974] B. Sz.-Nagy, C. Foias, Regular factorizations of contractions, Proc. Amer. Math. Soc. 43 (1974), 91-93.

[T1989] S. R. Treil, Geometric methods in spectral theory of vector-valued functions: some recent results, in: Toeplitz Operators and Spectral Functions Theory: Essays from the Leningrad Seminar on Operator Theory (ed. N. Nikolskii), pp. 209-280, Oper. Theory Adv. Appl. 42 Birkhäuser (Basel), 1989.

[Z1996] K. Zhu, Sub-Bergman Hilbert spaces on the unit disk. Indiana Univ. Math. J. 45 (1996), no. 1, 165-176.

[Z2003] K. Zhu, Sub-Bergman Hilbert spaces on the unit disk. II, J. Funct. Anal. 202 (2003), no. 2, 327-341.

Department of Mathematics, Virginia Tech, Blacksburg, VA 240610123, USA

E-mail address: joball@math.vt.edu

Department of Mathematics, The College of William and Mary, WiLliamsBurg VA 23187-8795, USA

E-mail address: vladi@math.wm.edu 